\title{
ON MODULARITY OF RIGID AND NONRIGID CALABI-YAU VARIETIES ASSOCIATED TO THE ROOT LATTICE $A_{4}$
}

\author{
KLAUS HULEK AND HELENA VERRILL
}

\begin{abstract}
We prove the modularity of four rigid and three nonrigid CalabiYau threefolds associated with the $A_{4}$ root lattice.
\end{abstract}

\section{$\S 1$. Introduction}

In this paper we investigate the geometry and arithmetic of a family of Calabi-Yau threefolds $X_{\boldsymbol{a}}, \boldsymbol{a}=\left(a_{1}: \cdots: a_{6}\right) \in \mathbb{P}^{5}$, birational to the projective hypersurface in $T:=\mathbb{P}^{4} \backslash\left\{X_{1} \cdots X_{5}=0\right\}$ given by

$$
X_{\boldsymbol{a}} \cap T:\left(X_{1}+\cdots+X_{5}\right)\left(\frac{a_{1}}{X_{1}}+\cdots+\frac{a_{5}}{X_{5}}\right)=a_{6} .
$$

Our motivation is to find further examples of modular Calabi-Yau varieties, i.e., of Calabi-Yau varieties which are defined over the rationals and whose $L$-series can be described in terms of modular forms. This is motivated by the Fontaine-Mazur conjecture on the modularity of two dimensional $\ell$-adic Galois representations coming from geometry [FM], which is a generalization of the Taniyama-Shimura-Weil conjecture on the modularity of elliptic curves, proved by Wiles et al, [Wi], [BCDT]. More precisely, Fontaine and Mazur [FM] define the notions of a "geometric Galois representation", and a "Galois representation coming from geometry". They conjecture that geometric Galois representations are precisely the Galois representations coming from geometry (such as the ones we consider) ([FM, Conjecture 1]), and combining this with classical conjectures (see e.g. [Se2]) leads them to the conjecture that two-dimensional irreducible geometric Galois representations are modular up to a Tate twist ([FM, Conjecture 3c] $]$ ). From these two conjectures, one obtains the conjecture that two dimensional irreducible Galois representations coming from geometry are modular up to a

Received August 18, 2003.

2000 Mathematics Subject Classification: 14J32, 14G25, 11F03, 11F23, 11 F32. 
Tate twist. We will use the term "modular" to mean "modular up to a Tate twist", and even to denote direct sums of modular Galois representations.

Rigid Calabi-Yau threefolds (defined over $\mathbb{Q}$ ) are expected to be modular, since they have 2-dimensional middle cohomology. One expects the $L$-series of the Galois action on the middle $\ell$-adic cohomology to be the Mellin transform of a weight 4 elliptic modular form. Although recently Dieulefait and Manoharmayum [DM] proved that a rigid Calabi-Yau threefold is modular provided it has good reduction at 3 and 7 , or at 5 and another suitable prime (in fact most of our examples have bad reduction at 3 and 5, so this result does not apply, and in general does not determine the exact modular form), it is still the case that relatively few examples of modular Calabi-Yau threefolds are explicitly known. Most currently known examples are given in Yui's survey articles [Y1], [Y2]. Other recent examples are given by $[\mathrm{CM}]$.

Modularity has been also conjectured for certain nonrigid examples, e.g., $[\mathrm{CM}]$. What is new in this paper is the proof of modularity for several nonrigid examples. Note that we mean modularity in the sense of FontaineMazur, i.e., the semisimplification of the Galois representation is a sum of 2 dimensional pieces. There are few examples of other kinds of modularity of nonrigid Calabi-Yau threefolds known. Consani and Scholten [CS] consider an example corresponding to a Hilbert modular form for which they provide evidence for the modularity and Livné and Yui [LY] very recently gave some cases involving weight 2 and 3 forms. Their examples and techniques are quite different from ours.

We shall study a certain 5-dimensional family $X_{\boldsymbol{a}}, \boldsymbol{a} \in \mathbb{P}^{5}$, of (singular) Calabi-Yau threefolds, associated to the root lattice $A_{4}$, by means of Batyrev's construction [Ba] of Calabi-Yau varieties as toric hypersurfaces.

The rigid cases are $X_{1}, X_{9}, X_{(1: 1: 1: 1: 4: 4)}$, and $X_{(1: 1: 1: 4: 4: 9)}$, where $X_{t}:=$ $X_{(1: 1: 1: 1: 1: t)}$. These have 40, 35, 37 and 35 nodes respectively, and their (big) resolutions have 2-dimensional middle cohomology. We will show that the $L$-series is the Mellin transform of a modular form of weight 4 , and level 6 , 6, 12 and 60 respectively. The first few terms of their $q$-expansions are

$$
\begin{aligned}
f_{6} & =q-2 q^{2}-3 q^{3}+4 q^{4}+6 q^{5}+6 q^{6}-16 q^{7}-8 q^{8}+\cdots, \\
f_{12} & =q+3 q^{3}-18 q^{5}+8 q^{7}+9 q^{9}+36 q^{11}-10 q^{13}+\cdots \\
f_{60} & =q-3 q^{3}-5 q^{5}-28 q^{7}+9 q^{9}-24 q^{11}-70 q^{13}+15 q^{15}+\cdots,
\end{aligned}
$$

where $f_{N}$ has level $N$. Although the middle cohomology of $X_{1}$ and $X_{9}$ have the same $L$-series, we will see that they are not birational to each other, 
though by a conjecture of Tate, one expects a correspondence between them. The nonrigid examples we consider are $X_{25}, X_{(1: 1: 1: 9: 9: 9)}$ and $X_{(1: 1: 4: 4: 4: 16)}$. In these cases we show that $L$-series of the middle cohomology of the big resolutions are

$$
L\left(f_{30}, s\right) L\left(g_{30}, s-1\right)^{4}, \quad L\left(f_{30}^{\prime}, s\right) L\left(g_{30}, s-1\right)^{2}, \quad L\left(f_{90}, s\right) L\left(g_{30}, s-1\right)
$$

respectively, where $L(h)$ denotes the Mellin transform of the function $h$, and the functions $g_{30}, f_{30}, f_{30}^{\prime}$ and $f_{90}$ are cuspidal Hecke eigen newforms, $g_{30}$ having weight 2 , the others weight 4 , and $f_{90}$ having level 90 , the others level 30. (The level 30 has, to our knowledge, not previously appeared in examples of this kind.) The first few terms of the $q$-expansions are

(6) $f_{30}(q)=q-2 q^{2}+3 q^{3}+4 q^{4}+5 q^{5}-6 q^{6}+32 q^{7}-8 q^{8}+9 q^{9}+\cdots$

(7) $f_{30}^{\prime}(q)=q+2 q^{2}+3 q^{3}+4 q^{4}-5 q^{5}+6 q^{6}-4 q^{7}+8 q^{8}+9 q^{9}+\cdots$

(8) $f_{90}(q)=q-2 q^{2}+4 q^{4}-5 q^{5}-4 q^{7}-8 q^{8}+10 q^{10}-12 q^{11}+\cdots$

Given expression (4), one would expect, by the Tate conjecture, that there is a geometric reason for the occurrence of the weight 2 modular form $g_{30}$, which is the Mellin transform of the $L$-series of a certain elliptic curve. We will see that this is indeed the case.

In both rigid and nonrigid cases, we use the powerful theorem due to Faltings, Serre and Livné [Li], which permits one to determine 2-dimensional Galois representations from a finite set of data. In practice this means counting the number of points modulo $p$ for a given finite number of primes, a task which can be done easily by computer.

In Section 2 we consider the toric geometry set up. In Section 3 we discuss the resolution of singularities of the singular subfamily $X_{\boldsymbol{a}}$. In Section 4 we show that $X_{\boldsymbol{a}}$ is birational to a fibre product of families of elliptic curves, which allows us to apply results of Schoen [Sc]. In Section 5 we study a certain elliptic surfaces contained in $X_{\boldsymbol{a}}$, and in Section 6 we count points and apply Livné's method to determine the $L$-series of the 7 cases of $X_{\boldsymbol{a}}$ mentioned above.

Figure 1 gives a schematic diagram of the 5-dimensional family $X_{\boldsymbol{a}}$ which we shall study, and some of its subfamilies (a complete list is given in Table 1). The diagram gives the dimension of these subfamilies and the value of $h^{12}$ of the big resolution $\widetilde{X}_{\boldsymbol{a}}$ of the general member of the 


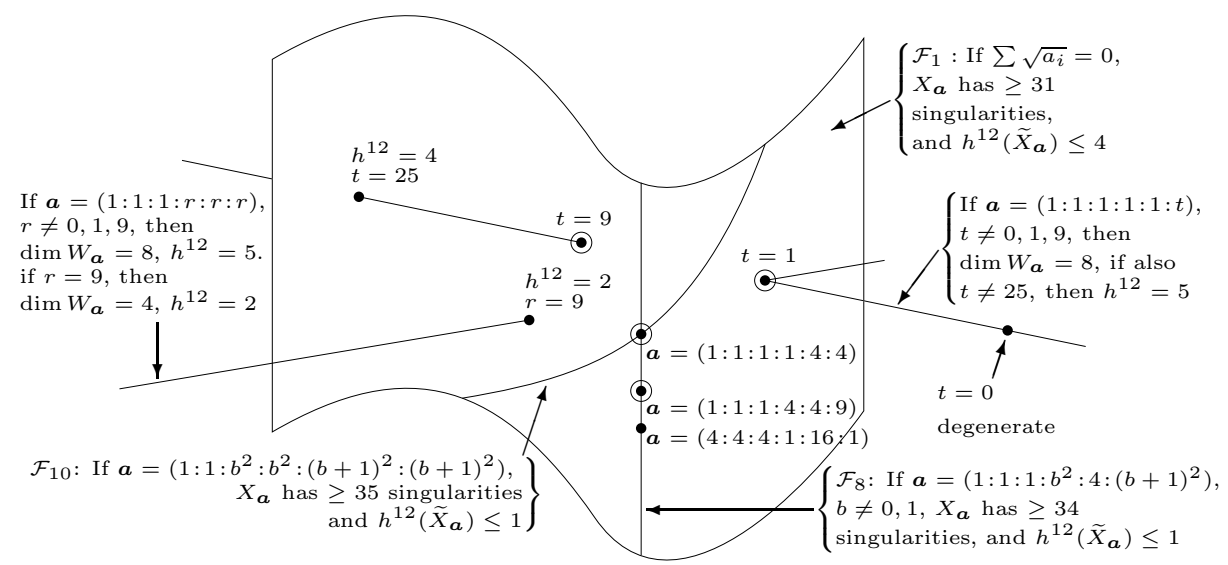

Figure 1: Values of the parameter $\boldsymbol{a}$ for certain members and subfamilies of the family of Calabi-Yau threefolds $X_{\boldsymbol{a}}$, with $\boldsymbol{a}$ for modular $X_{\boldsymbol{a}}$ marked by a point, which is circled if $X_{\boldsymbol{a}}$ is rigid.

subfamily. Values of $\boldsymbol{a}$ where $\tilde{X}_{\boldsymbol{a}}$ is modular are marked with points, and those which are rigid with circled points. In Section 5 we will see that for two equal indices $a_{i}=a_{j}$, there is a corresponding elliptic surface in $X_{\boldsymbol{a}}$. We call the piece of $H^{3}$ corresponding to these elliptic surfaces $W_{\boldsymbol{a}}$. When $\operatorname{dim} H^{3}-\operatorname{dim} W_{\boldsymbol{a}}=2$, i.e., $2 h^{12}=\operatorname{dim} W_{\boldsymbol{a}}$, one expects $X_{\boldsymbol{a}}$ to be modular, which we will see is the case for all 7 marked points in the diagram.

Finally we would like to point out that the family of Calabi-Yau varieties which we are considering in this paper has recently also appeared in a different context. C. Borcea has studied these varieties in the context of configuration spaces of planar polygons (see [Bo] where these varieties are called Darboux varieties).

Acknowledgements. We are grateful to the following institutions for support: to the DFG for grant $\mathrm{Hu} 337 / 5-1$ (Schwerpunktprogramm "Globale Methoden in der komplexen Geometrie") and to the University of Essen and M. Levine for hospitality during a stay supported by a Wolfgang Paul stipend. We are also greatly indebted to V. Batyrev, W. Fulton, J. Kollár and P. H. M. Wilson whose comments on intersection theory and Calabi-Yau manifolds were very helpful. We also thank N. Fakhruddin for useful remarks on $\ell$-adic Galois representations and M. Schütt for pointing out some misprints. 
Notation. In this paper we consider projective Calabi-Yau varieties defined by polynomial equations with coefficients in $\mathbb{Z}$. We work over the field $k$, where $k=\mathbb{C}, \mathbb{Q}, \overline{\mathbb{Q}}, \mathbb{F}_{p}$ or $\overline{\mathbb{F}}_{p}$. Further notation is as follows.

$M_{A_{4}}$ The $A_{4}$ root lattice, as a sublattice of $\mathbb{Z}^{5}$.

$N_{A_{4}} \quad\left(M_{A_{4}}\right)^{\vee}$, identified with a sublattice of $M_{A_{4}} \otimes \mathbb{Q}$.

$\varepsilon_{i j} \quad$ Point in $M_{A_{4}}$ at $e_{i}-e_{j}$.

$\Delta_{A_{4}} \quad$ Polytope in $M_{A_{4}} \otimes \mathbb{R}$ with vertices at $\varepsilon_{i j}$.

$\widetilde{\Sigma}_{A_{4}}$ Fan in $N_{A_{4}} \otimes \mathbb{R}$ given by all faces of the Weyl chambers.

$\widetilde{\Sigma}^{3}$ 3-dimensional cones in $\widetilde{\Sigma}_{A_{4}}$.

$\widetilde{P} \quad$ Smooth toric variety defined by $\widetilde{\Sigma}_{A_{4}}$.

$\mathbb{A}_{\sigma}^{4} \quad$ Affine piece of $\widetilde{P}$ corresponding to $\sigma \in \widetilde{\Sigma}^{3}$.

$T \quad$ Torus $\left(k^{*}\right)^{4} \subset \widetilde{P}$.

$T_{\sigma} \quad$ Orbit under the torus action corresponding to $\sigma \in \widetilde{\Sigma}_{A_{4}}$; $\widetilde{P}=\bigsqcup_{\sigma \in \widetilde{\Sigma}} T_{\sigma}$.

$X_{u} \quad$ For $u \in \mathbb{P}^{20}$, a Calabi-Yau threefold defined in $\widetilde{P}$ defined by $\Delta_{A_{4}}$.

$X_{\boldsymbol{a}} \quad$ For $\boldsymbol{a} \in \mathbb{P}^{5}$, a member of a 5 dimensional family of singular Calabi-Yaus in $\widetilde{P}$, with 30 nodes on $X_{\boldsymbol{a}} \backslash T$ if $\prod_{i=1}^{5} a_{i} \neq 0$.

$\bar{X}_{\boldsymbol{a}} \quad$ A Calabi-Yau given by taking a specific choice of small projective resolution of the 30 singularities on $X_{\boldsymbol{a}} \backslash T$.

$\widetilde{\bar{X}}_{\boldsymbol{a}} \quad$ Big resolution of remaining singularities on $\bar{X}_{\boldsymbol{a}}$.

$X_{t} \quad X_{(1: 1: 1: 1: 1: t)}$.

$\widetilde{X} \quad$ Big resolution of $X=X_{u}, X_{\boldsymbol{a}}, \bar{X}_{\boldsymbol{a}}$ or $X_{t}$, for $X$ irreducible.

$\widehat{X} \quad$ A choice of small projective resolution of $X$, if one exists.

\section{§. Toric varieties}

Batyrev [Ba] constructs Calabi-Yau varieties as hypersurfaces in a toric variety defined by a reflexive polytope $\Delta$ ([Ba, p. 510]), in a lattice $M$, as follows.

The pair $(M, \Delta)$ gives rise to a fan $\Sigma$ in the dual space $N_{\mathbb{R}}=M_{\mathbb{R}}^{\vee}$, and a strictly convex support function $h$ on $N_{\mathbb{R}}$. Let $\left(P, \mathcal{O}_{P}(1)\right)$ be the corresponding polarized toric variety. In general $P$ is a singular Fano variety with Gorenstein singularities and $\mathcal{O}_{P}(1)$ is the anticanonical bundle.

Batyrev shows that the general element in the anticanonical system is a Calabi-Yau variety, with canonical singularities exactly at the singular points of $P$. A desingularization $\widetilde{P}$ of $P$ can be constructed by taking the maximal projective triangulation $\widetilde{\Delta^{*}}$ of the dual polytope $\Delta^{*}$. We denote the corresponding fan by $\widetilde{\Sigma}$. The strict transforms of the anticanonical 


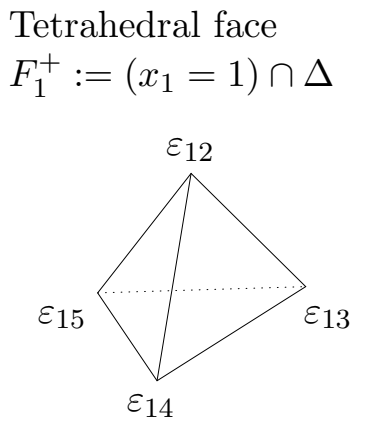

there are 10 such faces
Prism face

$F_{15}^{+}:=\left(x_{1}+x_{5}=1\right) \cap \Delta$

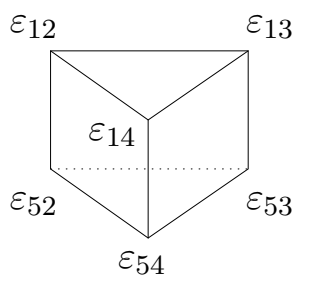

there are 20 such faces

Figure 2: Three dimensional faces of $\Delta$.

divisors on $P$ define a family of Calabi-Yau threefolds on $\widetilde{P}$ whose general element $X_{u}$ is smooth.

2.1. The polytopes $\Delta_{A_{4}}, \Delta_{A_{4}}^{*}$ and $\widetilde{\Delta_{A_{4}}^{*}}$

We now describe the lattice and polytope to which we apply Batyrev's construction. More generally, in the following construction one may replace $A_{4}$ by $A_{n}$, defined similarly; more details can be found in [DL], [P] and [Lu].

Let $M$ be the $A_{4}$ root lattice, given as the following sublattice of $\mathbb{Z}^{5}$ :

$$
M=M_{A_{4}}=\left\{\left(x_{1}, x_{2}, x_{3}, x_{4}, x_{5}\right) \mid x_{i} \in \mathbb{Z}, \sum x_{i}=0\right\} \subset \mathbb{Z}^{5} .
$$

The inner product on $M$ is induced by the standard inner product on $\mathbb{Z}^{5}$, and we identify the dual lattice $N:=\left(M_{A_{4}}\right)^{\vee}$ with a sublattice of $M_{\mathbb{R}}:=$ $M \otimes \mathbb{R}$.

Definition 2.1. The polytope $\Delta=\Delta_{A_{4}}$ in $M_{\mathbb{R}}$ is defined to be the convex hull of the roots $\varepsilon_{i j}:=e_{i}-e_{j}, 1 \leq i, j \leq 5, i \neq j$ of $M$, where $e_{1}, \ldots, e_{5}$ is the standard basis for $\mathbb{Z}^{5}$. In $[\mathrm{V}, \mathrm{p} .427]$ it is shown that $\Delta$ is reflexive.

It is a simple combinatorial exercise to enumerate the faces of $\Delta_{A_{4}}$, $\Delta_{A_{4}}^{*}$ and $\widetilde{\Delta_{A_{4}}^{*}}$. We have the following results.

Lemma 2.2. The polytope $\Delta$ has 20 vertices, 60 edges, 30 square faces, 40 triangular faces, and 30 three dimensional faces, given by

$$
F_{i}^{\varepsilon}:=\left(x_{i}=\varepsilon 1\right) \cap \Delta, \quad F_{i j}^{\varepsilon}:=\left(x_{i}+x_{j}=\varepsilon 1\right) \cap \Delta,
$$



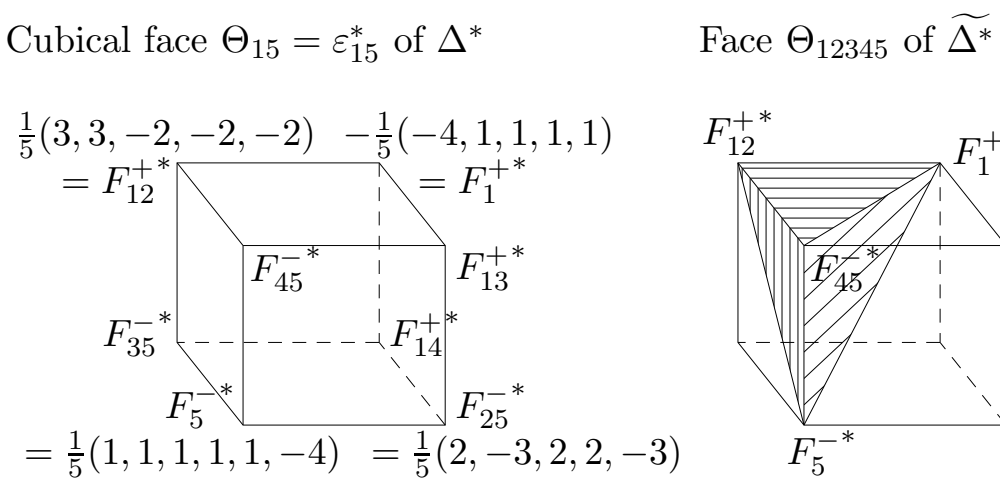

Figure 3: Three dimensional faces of $\Delta^{*}$ and $\widetilde{\Delta^{*}}$.

for $1 \leq i, j \leq 5, i \neq j$, and $\varepsilon= \pm$. Two of these faces are shown in Figure 2.

Proof. See [Lu, Lemma 1.18 and Korollar 1.19].

LEMMA 2.3. The dual polytope $\Delta^{*}$ has 30 vertices and 20 three dimensional cubical faces, $\Theta_{i j}$ for $1 \leq i, j \leq 5, i \neq j$. The vertices of $\Theta_{15}$ are shown in Figure 3, and $\Theta_{i j}=\sigma \Theta_{15}$, where $\sigma \in S_{5}$ with $\sigma: 1,5 \mapsto i, j$.

Proof. See [Lu, Lemma 1.46].

LEMMA 2.4. The subdivided polytope $\widetilde{\Delta^{*}}$ has 120 three dimensional faces, which are translations under $S_{5}$ of $\Theta_{12345}$ given in Figure 3.

Proof. See [Lu, Beispiel 2.34].

In order to apply Batyrev's formulae for $h^{11}$ and $h^{12}$ we need to count the number of lattice points in the interior of the faces of $\Delta$ and $\Delta^{*}$. We make use of the following easy result.

LEMMA 2.5. If $\mathbf{w}_{1}, \mathbf{w}_{2}, \mathbf{w}_{3}, \mathbf{w}_{4}$ is a basis for a lattice $L$, and $\Theta$ is a polytope with vertices $\mathbf{0}, \mathbf{w}_{1}, \ldots, \mathbf{w}_{4}$, then the only lattice points in $\Theta$ are its vertices.

By taking appropriate subdivisions of the faces $F_{i j}^{\varepsilon}$ and $\Theta_{i j}$, we have

LEMMA 2.6. No proper face of $\Delta$ or $\Delta^{*}$ contains an interior lattice point, and the only lattice point in the interior of $\Delta$ or $\Delta^{*}$ is the origin. 


\subsection{The toric variety $\widetilde{P}$ defined by the fan $\widetilde{\Sigma}$}

The fan $\widetilde{\Sigma}$ in $N_{\mathbb{R}}$ consists of cones given by the 120 Weyl chambers $\sigma_{i j k l m}=\left\{\left(\alpha_{1}, \alpha_{2}, \alpha_{3}, \alpha_{4}, \alpha_{5}\right) \in \mathbb{R}^{5} \mid \sum_{v=1}^{5} \alpha_{v}=0, \alpha_{i} \geq \alpha_{j} \geq \alpha_{k} \geq \alpha_{l} \geq \alpha_{m}\right\}$,

where $\{i, j, k, l, m\}=\{1,2,3,4,5\}$, together with all their subfaces. E.g., $\sigma_{12345}$ is the cone on $\Theta_{12345}$ (see Figure 3). The dual cones are given by

$$
\sigma_{i j k l m}^{\vee}:=\mathbb{R}_{\geq 0}\left(e_{i}-e_{j}\right)+\mathbb{R}_{\geq 0}\left(e_{j}-e_{k}\right)+\mathbb{R}_{\geq 0}\left(e_{k}-e_{l}\right)+\mathbb{R}_{\geq 0}\left(e_{l}-e_{m}\right) .
$$

We will consider Calabi-Yau threefold hypersurfaces in the toric variety $\widetilde{P}$ defined by $\widetilde{\Sigma}$. We first fix choices of local and global coordinates for $\widetilde{P}$.

We identify the torus $T \cong\left(k^{*}\right)^{4} \subset \widetilde{P}$ with $\mathbb{P}^{4} \backslash\left(\prod_{i=1}^{5} X_{i}=0\right)$, and use the projective coordinates $X_{1}, \ldots, X_{5}$ of $\mathbb{P}^{4}$ when considering points in $T$.

For the affine piece $\mathbb{A}_{\varsigma}^{4}:=\operatorname{Spec}\left(k\left[\varsigma\left(\sigma_{12345}^{\vee}\right)\right]\right) \subset \widetilde{P}$, where $\varsigma \in S_{5}$, we use coordinates $x_{\varsigma}, y_{\varsigma}, z_{\varsigma}, w_{\varsigma}$ corresponding to the basis $\varepsilon_{\varsigma(12)}, \varepsilon_{\varsigma(23)}, \varepsilon_{\varsigma(34)}$, $\varepsilon_{\varsigma}(45)$ of $\varsigma\left(\sigma_{12345}^{\vee}\right)$. Usually we just write $x, y, z, w$.

The identification of $T$ and $\mathbb{A}_{\varsigma}^{4} \backslash\left(x_{\varsigma} y_{\varsigma} z_{\varsigma} w_{\varsigma}=0\right)$ is given by

$$
\begin{array}{rlrl}
x_{\varsigma} & =X_{\varsigma(1)} / X_{\varsigma(2)}, & y_{\varsigma} & =X_{\varsigma(2)} / X_{\varsigma(3)}, \\
z_{\varsigma} & =X_{\varsigma(3)} / X_{\varsigma(4)}, & w_{\varsigma}=X_{\varsigma(4)} / X_{\varsigma(5)} .
\end{array}
$$

This relationship between the coordinates of $\mathbb{P}^{4}$ and of the affine pieces of $\widetilde{P}$ is explained by the following lemma.

Lemma 2.7. The variety $\widetilde{P}$ is the graph of the Cremona transformation $X_{i} \mapsto 1 / X_{i}$ of $\mathbb{P}^{4}$. Thus $\widetilde{P}$ is obtained from $\mathbb{P}^{4}$ by blowing up successively the (strict transforms of the) points $(1: 0: 0: 0),(0: 1: 0$ : $0), \ldots,(0: 0: 0: 1)$, lines and planes spanned by any subset of these points.

Proof. See [DL, Lemma 5.1].

2.2.1. Toric orbits in $\widetilde{P}$

There is a decomposition $\widetilde{P}=\bigsqcup_{\sigma \in \widetilde{\Sigma}} T_{\sigma}$, where $T_{\sigma}$ is the toric orbit of $\widetilde{P}$ corresponding to $\sigma \in \widetilde{\Sigma}$. Since $\widetilde{\Sigma}$ is given by taking cones on the faces of $\widetilde{\Delta}^{*}$, we use the notation $T_{\Theta}:=T_{\mathbb{R}_{+} \Theta}$ where $\Theta$ is a face of $\widetilde{\Delta}^{*}$. By standard methods of toric geometry we have

$$
\begin{aligned}
& \overline{T_{F_{i}^{\varepsilon *}}} \cong \widetilde{P}_{A_{3}}, \\
& \overline{T_{F_{i j}^{\varepsilon *}}} \cong \widetilde{P}_{A_{2}} \times \widetilde{P}_{A_{1}} \cong \widetilde{\mathbb{P}}^{2} \times \mathbb{P}^{1},
\end{aligned}
$$




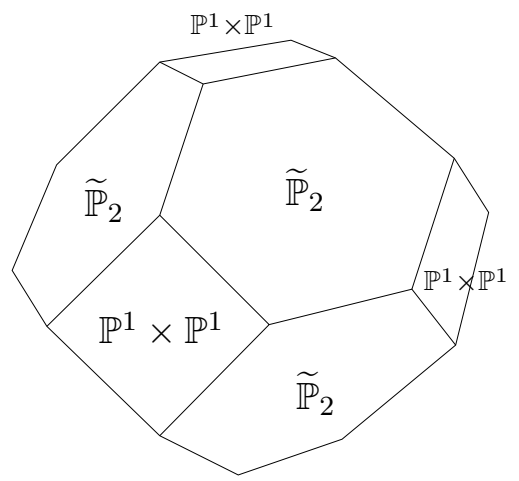

There are 10 copies of $\overline{T_{F_{i}^{\varepsilon *}}}$ in $\widetilde{P} \backslash T$, two being $\overline{\{x=0\}}$ and $\overline{\{w=0\}}$. The closures of $T_{\Theta^{*}}$, for $\Theta$ a two dimensional face of $F_{j}^{\varepsilon}$, consists of 8 copies of $\widetilde{\mathbb{P}}^{2}$ and 6 copies of $\mathbb{P}^{1} \times \mathbb{P}^{1}$. These intersect as indicated in this figure; hexagonal faces correspond to $\widetilde{\mathbb{P}}^{2}$, and square faces correspond to $\mathbb{P}^{1} \times \mathbb{P}^{1}$.

Figure 4: The threefold $\overline{T_{F_{i}^{\varepsilon *}}}$ in $\widetilde{P} \backslash T$.

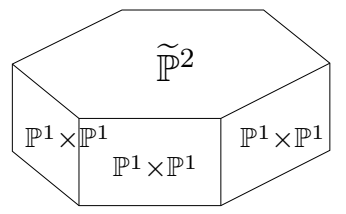

There are 20 copies of $\overline{T_{F_{i j}^{*}}{ }^{*}}$ in $\widetilde{P}$, two being $\overline{\{y=0\}}$ and $\overline{\{z=0\}}$. The closures of $T_{\Theta^{*}}$, for $\Theta$ a two dimensional face of $F_{i j}^{\varepsilon}$, consists of 2 copies of $\widetilde{\mathbb{P}}^{2}$, and 6 copies of $\mathbb{P}^{1} \times \mathbb{P}^{1}$, corresponding to the hexagons and squares respectively in this figure.

Figure 5: The threefold $\overline{T_{F_{i j}^{\varepsilon}}}$ in $\widetilde{P} \backslash T$.

where $\widetilde{P}_{A_{n}}$ is the toric variety corresponding to the root lattice $A_{n}$, and $\widetilde{\mathbb{P}}^{2}$ is $\mathbb{P}^{2}$ blown up in 3 points. These are sketched in Figures 4 and 5 .

In terms of local coordinates $x, y, z, w$ for $\mathbb{A}_{\text {id }}^{4}$, we have

$$
\begin{aligned}
& T_{F_{4}^{-*}}=T_{\frac{1}{5}(1,1,1,1,-4)}=\{x=0 \neq y z w\}, \\
& T_{F_{45}^{-*}}=T_{\frac{1}{5}(2,2,2,-3,-3)}=\{y=0 \neq x z w\} \text {, } \\
& T_{F_{12}^{+*}}=T_{\frac{1}{5}(3,3,-2,-2,-2)}=\{z=0 \neq x y w\} \text {, } \\
& T_{F_{1}^{+*}}=T_{\frac{1}{5}(4,-1,-1,-1,-1)}=\{w=0 \neq x y z\} \text {. }
\end{aligned}
$$

The intersections of the closures these hypersurfaces is sketched in Figure 6.

\section{§3. The Calabi-Yau varieties}

Following Batyrev, we define a family of hypersurfaces in $\widetilde{P}$, given by elements $X \in\left|-K_{\widetilde{P}}\right|$. The general member of the family is given by 


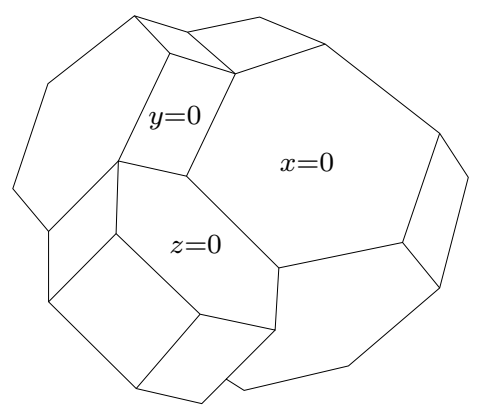

The closures of $\{x=0\},\{y=0\}$ and $\{z=0\}$ intersect as indicated by the intersections of the corresponding polyhedra. A polyhedron corresponding to $\{w=0\}$ meets these polyhedra in the labeled faces. Where two polyhedra meet in a hexagon the corresponding threefolds have intersection $\widetilde{\mathbb{P}}^{2}$, and where they meet in a square the corresponding threefolds intersect in $\mathbb{P}^{1} \times \mathbb{P}^{1}$.

Figure 6: How $\overline{\{x=0\}}, \overline{\{y=0\}}$ and $\overline{\{z=0\}} \subset \widetilde{P} \backslash T$ meet.

an equation containing exactly the monomials corresponding to the lattice points of $\Delta$.

In our case, $\Delta$ has 21 lattice points, and so gives a 20 dimensional family. The general member, when restricted to the open torus $T$, has an equation

(9) $X_{u}: \sum_{1 \leq i, j \leq 5, i \neq j} u_{i j} X_{i} X_{j}^{-1}=t \quad$ for $u=\left(u_{12}: u_{13}: \cdots: u_{45}: t\right) \in \mathbb{P}^{20}$

in terms of the homogenous coordinates for $T \subset \mathbb{P}^{4}$.

Given the above analysis of $\Delta$ and $\Delta^{*}$, we can now prove the following.

Proposition 3.1. For every smooth member $X_{u}$ of the family of Calabi-Yau threefolds (9), we have

(i) The Euler number $e\left(X_{u}\right)=20$.

(ii) The Hodge numbers of $X_{u}$ are given by $h^{00}=h^{33}=h^{30}=h^{03}=1$, $h^{10}=h^{01}=h^{20}=h^{02}=0, h^{11}=26$ and $h^{12}=h^{21}=16$.

Proof. Since $X_{u}$ is smooth and Calabi-Yau the only Hodge numbers to be computed are $h^{11}$ and $h^{21}$. By [Ba, p. 521] we have

$$
\begin{aligned}
& h^{11}\left(X_{u}\right)=l\left(\Delta^{*}\right)-5-\sum_{\operatorname{codim} \Theta^{*}=1} l^{*}\left(\Theta^{*}\right)+\sum_{\operatorname{codim} \Theta^{*}=2} l^{*}\left(\Theta^{*}\right) l^{*}(\Theta), \\
& h^{21}\left(X_{u}\right)=l(\Delta)-5-\sum_{\operatorname{codim} \Theta=1} l^{*}(\Theta)+\sum_{\operatorname{codim} \Theta=2} l^{*}(\Theta) l^{*}\left(\Theta^{*}\right),
\end{aligned}
$$


where $l(\Theta)$ denotes the number of lattice points in $\Theta$ and $l^{*}(\Theta)$ denotes the number of interior lattice points of $\Theta$, for any face $\Theta$ of $\Delta$.

Lemmas 2.2, 2.3 and 2.6 imply that $l(\Delta)=21, l\left(\Delta^{*}\right)=31, l^{*}\left(\Delta^{*}\right)=1$ and $l^{*}\left(\Theta^{*}\right)=0$ for all proper faces $\Theta$ of $\Delta^{*}$. Hence $h^{11}\left(X_{u}\right)=26, h^{21}\left(X_{u}\right)=$ 16. The Euler characteristic is then given by $e\left(X_{u}\right)=2 h^{11}-2 h^{12}=20$.

\subsection{Resolution of singular Calabi-Yau threefolds}

We consider elements $X \in\left|-K_{\widetilde{P}}\right|$ which have $s$ nodes, but no other singularities. We denote the big resolution of $X$, obtained by blowing up the nodes, by $\widetilde{X}$. We also have $2^{s}$ small resolutions of $X$, where each node is replaced by a $\mathbb{P}^{1}$. It is not clear whether there are any small projective resolutions as these could all contain null homologous lines. By $\widehat{X}$ we denote a small projective resolution, when one exists.

Let $X_{u}$ be a smooth member of the family (9), and let $X_{\boldsymbol{a}}$ be an element of the family with $s$ nodes, but no other singularities. Then we have

Proposition 3.2. Let $\tilde{h}^{p q}=h^{p q}\left(\widetilde{X}_{\boldsymbol{a}}\right)$, resp. $\hat{h}^{p q}=h^{p q}\left(\widehat{X}_{\boldsymbol{a}}\right)$ be the Hodge numbers of the big resolution $\widetilde{X}_{\boldsymbol{a}}$ of $X_{\boldsymbol{a}}$, resp. a small projective resolution of $X_{\boldsymbol{a}}$. Then the following holds

(i) $e\left(X_{\boldsymbol{a}}\right)=e\left(X_{u}\right)+s, e\left(\widehat{X}_{\boldsymbol{a}}\right)=e\left(X_{u}\right)+2 s, e\left(\tilde{X}_{\boldsymbol{a}}\right)=e\left(X_{u}\right)+4 s$

(ii) $\tilde{h}^{30}=\tilde{h}^{03}=\hat{h}^{30}=\hat{h}^{03}=1$

(iii) $\tilde{h}^{10}=\tilde{h}^{01}=\hat{h}^{10}=\hat{h}^{01}=0, \tilde{h}^{20}=\tilde{h}^{02}=\hat{h}^{20}=\hat{h}^{02}=0$

(iv) $\tilde{h}^{11}-\tilde{h}^{12}=\frac{1}{2} e\left(\widetilde{X}_{\boldsymbol{a}}\right), \hat{h}^{11}-\hat{h}^{12}=\frac{1}{2} e\left(\widehat{X}_{\boldsymbol{a}}\right)$.

Proof. (i) These formulae are well known, cf. [C, Section 1] or [We, Kapitel II].

(ii) Since $h^{p 0}=h^{0 p}$ are birational invariants, it is enough to prove the assertion for $\tilde{h}^{30}$. Let $Q_{1}, \ldots, Q_{s}$ be the exceptional quadrics in $\widetilde{X}_{\boldsymbol{a}}$ and note that their normal bundle is $(-1,-1)$. Since $X_{\boldsymbol{a}}$ is a Calabi-Yau variety with $s$ nodes it follows that $\omega_{\widetilde{X}_{\boldsymbol{a}}}=\mathcal{O}_{\widetilde{X}_{\boldsymbol{a}}}\left(\sum_{i=1}^{s} Q_{i}\right)$ and $\tilde{h}^{30}=h^{0}\left(\omega_{\tilde{X}_{\boldsymbol{a}}}\right)=1$.

(iii) We consider the sequence

$$
0 \longrightarrow \mathcal{O}_{\widetilde{P}}\left(-K_{\widetilde{P}}\right) \longrightarrow \mathcal{O}_{\widetilde{P}} \longrightarrow \mathcal{O}_{X_{a}} \longrightarrow 0
$$

Since $h^{1}\left(\mathcal{O}_{\widetilde{P}}\right)=0$ and $h^{2}\left(\mathcal{O}_{\widetilde{P}}\left(-K_{\widetilde{P}}\right)\right)=h^{2}\left(\mathcal{O}_{\widetilde{P}}\right)=0$, we have $h^{1}\left(\mathcal{O}_{X_{\boldsymbol{a}}}\right)=0$. The resolution $\pi: \widetilde{X}_{\boldsymbol{a}} \rightarrow X_{\boldsymbol{a}}$ is the blow up of double points, i.e., of rational singularities, and hence $R^{1} \pi_{*} \mathcal{O}_{\widetilde{X}_{a}}=0$. By the Leray spectral sequence for 
the resolution this implies that $h^{1}\left(\mathcal{O}_{\widetilde{X}_{a}}\right)=h^{1}\left(\mathcal{O}_{X_{a}}\right)=0$, and hence, since these numbers are birational invariants, $\tilde{h}^{10}=\tilde{h}^{01}=\hat{h}^{10}=\hat{h}^{01}=0$.

It remains to prove that $\tilde{h}^{02}=h^{2}\left(\mathcal{O}_{\widetilde{X}_{\boldsymbol{a}}}\right)=h^{1}\left(\omega_{\widetilde{X}_{\boldsymbol{a}}}\right)=0$. The latter can be deduced from the exact sequence

$$
0 \longrightarrow \mathcal{O}_{\widetilde{X}_{\boldsymbol{a}}} \longrightarrow \omega_{\widetilde{X}_{\boldsymbol{a}}} \longrightarrow \bigoplus_{i=1}^{s} \mathcal{O}_{Q_{i}}(-1,-1) \longrightarrow 0
$$

together with the fact that $H^{1}\left(\mathcal{O}_{\widetilde{X}_{a}}\right)=0$ which we have already seen.

(iv) This follows immediately from (ii) and (iii).

\subsection{A five dimensional subfamily $X_{a}$ with 30 nodes}

We now turn our attention to a certain subfamily of (9) of Calabi-Yau threefolds of the form

$$
\begin{aligned}
& X_{\boldsymbol{a}} \cap T:\left(X_{1}+\cdots+X_{5}\right)\left(\frac{a_{1}}{X_{1}}+\cdots+\frac{a_{5}}{X_{5}}\right)=a_{6}=t \\
& \qquad \quad \text { for } \boldsymbol{a}=\left(a_{1}: a_{2}: a_{3}: a_{4}: a_{5}: t\right) \in \mathbb{P}^{5} .
\end{aligned}
$$

The variety $X_{\boldsymbol{a}}$ is the closure of $X_{\boldsymbol{a}} \cap T$ in the toric variety $\widetilde{P}$. For $t \in \mathbb{C}$, we will also use the notation $X_{t}:=X_{(1: 1: 1: 1: 1: t)}$.

In terms of the local coordinates $x=X_{1} / X_{2}, y=X_{2} / X_{3}, z=X_{3} / X_{4}$, $w=X_{4} / X_{5}$ for $\mathbb{A}_{\text {id }}^{4}$, given in Section 2.2, the equation (10) for $X_{\boldsymbol{a}}$ becomes

$\left(a_{1}+a_{2} x+a_{3} x y+a_{4} x y z+a_{5} x y z w\right)(1+w+w z+w z y+w z y x)=a_{6} x y z w$.

Remark 3.3. If $\boldsymbol{a}=\left(a_{1}: \cdots: a_{6}\right) \in \mathbb{P}^{5}$ with $\prod_{i=1}^{6} a_{i}=0$, then $X_{\boldsymbol{a}}$ is not irreducible, and so in general we assume $\prod_{i=1}^{6} a_{i} \neq 0$.

Other local equations are given by permuting the $a_{i}$ appropriately. Although this equation is not symmetric in the $a_{i}$, we will now see that up to birational equivalence $a_{6}$ plays the same role as the other $a_{i}$.

Lemma 3.4. The variety $X_{\boldsymbol{a}}$ defined by (10) is birational to a variety in $\mathbb{P}^{5}$ defined by two equations:

$$
\sum_{i=1}^{6} \frac{a_{i}}{X_{i}}=\sum_{i=1}^{6} X_{i}=0
$$

Proof. This follows immediately from setting $X_{6}=-\sum_{i=1}^{5} X_{i}$. 
Corollary 3.5. For any permutation $\sigma \in S_{6}$, the varieties $X_{\boldsymbol{a}}$ and $X_{\sigma(\boldsymbol{a})}$ are birational.

Remark 3.6. The Barth-Nieto quintic $N_{5}$ is the variety in $\mathbb{P}^{5}$ defined by (12) with all $a_{i}=1$. A corollary of the above lemma is that

$$
X_{(1: 1: 1: 1: 1: 1)}=X_{1} \sim_{\text {bir }} N_{5}
$$

3.2.1. The singularities of $X_{\boldsymbol{a}}$ on $X_{\boldsymbol{a}} \cap T$

Lemma 3.7. For $\boldsymbol{a}=\left(a_{1}: a_{2}: a_{3}: a_{4}: a_{5}: t\right) \in \mathbb{P}^{5}$, with $t \neq 0$, the variety $X_{\boldsymbol{a}}$ (over any field) has a singularity at $\boldsymbol{b} \in T$ if and only if $\boldsymbol{a}=\phi(\boldsymbol{b})$ for some $\boldsymbol{b} \in T \subset \mathbb{P}^{4}$, where $\phi$ is the map

$$
\begin{aligned}
\phi: T & \longrightarrow \mathbb{P}^{5} \\
(a: b: c: d: e) & \longmapsto\left(a^{2}: b^{2}: c^{2}: d^{2}: e^{2}:(a+b+c+d+e)^{2}\right) .
\end{aligned}
$$

Proof. Writing (10) as $f \cdot g=t$, and differentiating with respect to $X_{i}$ gives

$$
g-\frac{a_{i}}{X_{i}^{2}} f=0, \quad i=1, \ldots, 5
$$

which implies that a singular point has the form $P=\left( \pm \sqrt{a_{1}}: \cdots: \pm \sqrt{a_{5}}\right)$. Substituting into (10), we obtain

$$
\left(\sqrt{a_{1}} \pm \sqrt{a_{2}} \pm \sqrt{a_{3}} \pm \sqrt{a_{4}} \pm \sqrt{a_{5}}\right)^{2}=t,
$$

and so $\boldsymbol{a}$ has the claimed form.

Proposition 3.8. The singularity $\boldsymbol{b}=\left(b_{1}: b_{2}: b_{3}: b_{4}: b_{5}\right) \in X_{\phi(\boldsymbol{b})} \cap$ $T \subset \mathbb{P}^{4}$ in Lemma 3.7 is an $A_{1}$ singularity.

Proof. Consider the affine cover of $T$ with coordinates $y_{i}=X_{i} / X_{5}-$ $b_{i} / b_{5}$ for $1 \leq i \leq 4$. In terms of these coordinates, the singularity is at $(0,0,0,0)$, and the equation for $X_{\phi(b)}$ is given by

$$
\begin{aligned}
& \left(b_{1}+b_{2}+b_{3}+b_{4}+b_{5}\right)\left(\frac{y_{1}^{2}}{b_{1}}+\frac{y_{2}^{2}}{b_{2}}+\frac{y_{3}^{2}}{b_{3}}+\frac{y_{4}^{2}}{b_{4}}\right) \\
& -\left(y_{1}+y_{2}+y_{3}+y_{4}\right)^{2}+\text { higher order terms }=0 .
\end{aligned}
$$


The matrix of the quadratic form given by the degree two part of (15) is

$$
M:=\left(\begin{array}{cccc}
s / b_{1}-1 & -1 & -1 & -1 \\
-1 & s / b_{2}-1 & -1 & -1 \\
-1 & -1 & s / b_{3}-1 & -1 \\
-1 & -1 & -1 & s / b_{4}-1
\end{array}\right),
$$

where $s=b_{1}+b_{2}+b_{3}+b_{4}+b_{5}$. We have

$$
\operatorname{det}(M)=\frac{b_{5}\left(b_{1}+b_{2}+b_{3}+b_{4}+b_{5}\right)^{3}}{b_{1} b_{2} b_{3} b_{4}},
$$

which is non zero since $\boldsymbol{b} \in T$. Thus the singularity is as claimed.

3.2.2. The singularities of $X_{\boldsymbol{a}}$ on $X_{\boldsymbol{a}} \backslash T$

Lemma 3.9. Let $\boldsymbol{a}=\left(a_{1}: \cdots: a_{6}\right) \in \mathbb{P}$ with all $a_{i} \neq 0$, and $\varsigma \in S_{5}$. Then over any field, $X_{\boldsymbol{a}}$ has singularities in $\left(X_{\boldsymbol{a}} \backslash T\right) \cap \mathbb{A}_{\varsigma}^{4}$ only at the point

$$
\left(x_{\varsigma}, y_{\varsigma}, z_{\varsigma}, w_{\varsigma}\right)=\left(-a_{\varsigma(1)} / a_{\varsigma(2)}, 0,0,-1\right),
$$

where $x_{\varsigma}, y_{\varsigma}, z_{\varsigma}, w_{\varsigma}$ are as in Section 2.2. This singularity is a node.

Proof. This follows easily by computing the partial derivatives of (11), and setting them to zero.

LEMma 3.10. For general $\boldsymbol{a} \in \mathbb{P}^{5}$ there are exactly 30 singularities on $X_{a}$.

Proof. By Lemma 3.7, in general, $X_{\boldsymbol{a}} \cap T$ is smooth. By Lemma 3.9, there is only one singularity in the affine piece $\mathbb{A}_{\varsigma}^{4}$, contained in $\left(y_{\varsigma}=z_{\varsigma}=\right.$

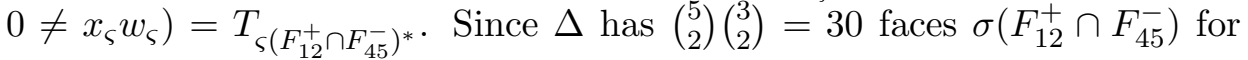
$\sigma \in S_{5}$, this implies the result.

\subsubsection{Classification of singular subfamilies}

By Lemma 3.7, to find the number of singularities on $X_{\boldsymbol{a}} \cap T$, we need to determine the number of $\boldsymbol{b}$ with $\boldsymbol{a}=\phi(\boldsymbol{b})$. We obtain the following result.

Proposition 3.11. For $\boldsymbol{b}=\left(b_{1}: \cdots: b_{5}\right) \in T$, the number of nodes on $X_{\phi}(\boldsymbol{b})$ is given by

$$
30+\#\left\{J: J \subset\{1,2,3,4,5\}, \sum_{i \in J} b_{i}=0\right\} .
$$


Proof. Lemma 3.10 gives 30 nodes on $X_{\boldsymbol{a}} \backslash T$. By Lemma 3.7, the only nodes on $X_{\boldsymbol{a}} \cap T$ are at $\boldsymbol{c}$ with $\phi(\boldsymbol{c})=\phi(\boldsymbol{b})$. Let $\boldsymbol{c}=\left(c_{1}: \cdots: c_{5}\right) \in \mathbb{P}^{4}$. After scaling, $b_{i}=\varepsilon_{i} c_{i}$, with $\varepsilon_{i}= \pm 1$ and $\sum b_{i}=\sum c_{i}$. From this we obtain

$$
\sum\left(1-\varepsilon_{i}\right) b_{i}=0
$$

Thus the correspondence between nodes in $T$ and subsets of $\{1,2,3,4,5\}$ is given by

$$
c \longleftrightarrow\left\{i: c_{i} \neq b_{i}\right\}
$$

with the empty set corresponding to $\boldsymbol{b}$.

Remark 3.12. By considering how subsets of the $b_{i}$ can intersect, this lemma allows us to classify all subfamilies of $X_{\boldsymbol{a}}$ with more than 30 nodes. These are given in Table 1 . In this table, sets $\mathcal{F}_{i}$ are given in a shortened form, e.g., $\mathcal{F}_{7}=\phi\{(a: a: a:-a: b)\}$ should be read as

$$
\mathcal{F}_{7}:=\phi\left\{(a: a: a:-a: b) \in \mathbb{P}^{4} \mid a, b \in k \backslash\{0\}\right\} .
$$

To use this table, one must take the smallest set $\mathcal{F}_{i}$ containing a given $\boldsymbol{a}$, up to permutation of coordinates; e.g.; $(1: 1: 1: 1: 1: 1) \in \mathcal{F}_{15} \subset \mathcal{F}_{11} \subset$ $\mathcal{F}_{4} \subset \mathcal{F}_{2} \subset \mathcal{F}_{0}$; the data for $X_{1}$ is given by the last line of the table.

As a corollary of Proposition 3.11, we have the following result.

Proposition 3.13. The Euler numbers of $\widetilde{X}_{\boldsymbol{a}}, \widetilde{\bar{X}}_{\boldsymbol{a}}$, and of the small resolution $\widehat{X}_{\boldsymbol{a}}$, if it exists, are given in Table 1 .

Proof. This follows from Proposition 3.1(i) and Proposition 3.2(i).

We will discuss whether $\widehat{X}_{\boldsymbol{a}}$ exists in Sections 3.2.4, 3.2.7 and 4.1.

Remark 3.14. In Proposition 4.8 we will see that $h^{12}$ of the general member of any of the families in Table 1 is equal to the dimension of the family. In particular, we will see that for $\boldsymbol{a}$ in a zero dimensional family, $X_{a}$ is rigid. 


$\begin{array}{lrcccc}\text { Smallest family } \mathcal{F}_{i} & \text { dimension } & \text { number of } & e\left(\widetilde{X}_{\boldsymbol{a}}\right) & e\left(\widetilde{\widetilde{X}}_{\boldsymbol{a}}\right) & e\left(\widehat{X}_{\boldsymbol{a}}\right) \\ \text { containing } \boldsymbol{a} & \text { of } \mathcal{F}_{i} & \text { nodes on } X_{\boldsymbol{a}} & & & \\ \mathcal{F}_{0}=\left\{\boldsymbol{a} \in \mathbb{P}^{6}, a_{i} \neq 0\right\} & 5 & 30 & 140 & 80 & 80 \\ \mathcal{F}_{1}=\phi\{(a: b: c: d: e)\} & 4 & 30+1 & 144 & 84 & - \\ \mathcal{F}_{2}=\phi\{(a:-a: b: c: d)\} & 3 & 30+2 & 148 & 88 & - \\ \mathcal{F}_{3}=\phi\{(a: b:-a-b: c: d)\} & 3 & 30+2 & 148 & 88 & - \\ \mathcal{F}_{4}=\phi\{(a:-a: a: b: c)\} & 2 & 30+3 & 152 & 92 & - \\ \mathcal{F}_{5}=\phi\{(a:-a: b: a-b: c)\} & 2 & 30+3 & 152 & 92 & - \\ \mathcal{F}_{6}=\phi\{(a:-a: b:-b: c)\} & 2 & 30+4 & 156 & 96 & 88 \\ \mathcal{F}_{7}=\phi\{(a: a: a:-a: b)\} & 1 & 30+4 & 156 & 96 & - \\ \mathcal{F}_{8}=\phi\{(a: a: a:-2 a: b)\} & 1 & 30+4 & 156 & 96 & - \\ \mathcal{F}_{9}=\phi\{(a: a: b:-b: b-a)\} & 1 & 30+4 & 156 & 96 & - \\ \mathcal{F}_{10}=\phi\{(a: a: b: b:-a-b)\} & 1 & 30+5 & 160 & 100 & - \\ \mathcal{F}_{11}=\phi\{(a: a:-a:-a: b)\} & 1 & 30+6 & 164 & 104 & 92 \\ \mathcal{F}_{12}=\phi\{(1: 1: 1: 1:-1)\} & 0 & 30+5 & 160 & 100 & - \\ \mathcal{F}_{13}=\phi\{(1: 1: 1: 2:-2)\} & 0 & 30+5 & 160 & 100 & - \\ \mathcal{F}_{14}=\phi\{(1: 1: 1: 1:-2)\} & 0 & 30+7 & 168 & 108 & - \\ \mathcal{F}_{15}=\phi\{(1: 1: 1:-1:-1)\} & 0 & 30+10 & 180 & 120 & 100\end{array}$

Table 1: Number of nodes on $X_{\boldsymbol{a}}$ for subfamilies of $\boldsymbol{a}$, up to permutation of coordinates, as images of subfamilies of $\boldsymbol{b} \in T$, under $\phi$, given by (13); see Remark 3.12. The last 4 sets are $\{(1: 1: 1: 1: 1: 9)\},\{(1: 1: 1: 4:$ $4: 9)\},\{(1: 1: 1: 1: 4: 4)\}$ and $\{(1: 1: 1: 1: 1: 1)\}$ respectively.

\subsubsection{Weil divisors and small resolutions}

We shall now discuss whether $X_{\boldsymbol{a}}$ has a small projective resolution. If this is the case then $X_{\boldsymbol{a}}$ possesses a smooth projective Calabi-Yau model. We shall see later (Proposition 4.4) that these two statements are in fact equivalent.

First we recall a fact about small resolutions. Let $X$ be a projective variety, and $P \in X$ a double point. Near $P, X$ is locally analytically a cone over a quadric surface $Q$. The local divisor class group is generated by the cones $\Lambda_{1}, \Lambda_{2}$ over the rulings of $Q$. Blowing up $X$ in $P$ defines a (big) resolution $\pi: \widetilde{X} \rightarrow X$, with $\pi^{-1}(P) \cong Q$. Locally analytically we can also blow up $\Lambda_{1}$ and $\Lambda_{2}$, obtaining small resolutions $p_{i}: X_{i} \rightarrow X, i=1,2$, with $p_{i}^{-1}(P) \cong \mathbb{P}^{1}$. However, this is an analytic construction, and it is not clear 
whether the $X_{i}$ are projective. We have a commutative diagram

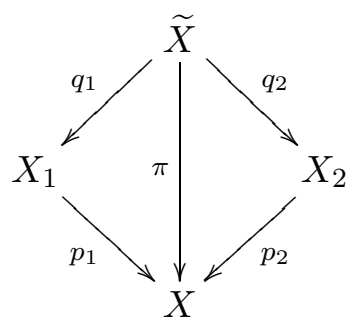

where the $q_{i}$ each blow down one family of rulings. This is Atiyah's flop. If $W$ is a global Weil divisor on $X$ through $P$, blowing up along $W$ gives a projective variety. If $W$ is not Cartier near $P$ then it is analytically locally equivalent to $a \Lambda_{1}$ or $a \Lambda_{2}$ for some $a>0$. By the universal property of the blow up, blowing up $X$ along $W$ is the same as performing a small resolution. For a discussion of this material see [EH, Example IV-27].

3.2.5. Small resolution $\bar{X}_{\boldsymbol{a}}$ of the 30 singularities on $X_{\boldsymbol{a}} \backslash T$

We now show that we can find a projective variety which is a small projective resolution of each of the 30 nodes on $X_{\boldsymbol{a}} \backslash T$. First we will define surfaces which define the Weil divisors we use for the blow up, as described above.

Definition 3.15. Let $1 \leq i<j \leq 5, i \neq j$ and $\varepsilon= \pm$. For some $\varsigma \in S_{5}$, we have $\overline{T_{F_{i j}^{\varepsilon *}}}=\overline{\left(y_{\varsigma}=0\right)}$ in the affine piece $\mathbb{A}_{\varsigma}^{4}$. We define the surface $S_{\boldsymbol{a}}^{\varepsilon i j}$ to be one of the two components of $\overline{\left(y_{\varsigma}=0\right)} \cap X_{\boldsymbol{a}}$, given in terms of the coordinates for $\mathbb{A}_{\varsigma}^{4}$ by

$$
S_{\boldsymbol{a}}^{\varepsilon i j}: \overline{\left(a_{\varsigma(1)}+a_{\varsigma(2)} x_{\varsigma}=y_{\varsigma}=0\right)}
$$

This is independent of which of the 6 possible $\varsigma$ is chosen. The surface $S_{\boldsymbol{a}}^{-45}$ is indicated in Figure 7.

Lemma 3.16. The 10 surfaces $S_{a}^{-i j}$ are smooth, disjoint, and each contains 3 nodes of $X_{\boldsymbol{a}}$.

Proof. Smoothness is clear. Set $T_{i j}:=T_{F_{i j}^{-*}}$. For $\{i, j\} \neq\{k, l\}$ the vertices $F_{i j}^{-*}$ and $F_{k l}^{-*}$ do not lie on a common edge of $\Delta^{*}$ (see Figure 3), so $\overline{T_{i j}} \cap \overline{T_{k l}}=\varnothing$, from which disjointness follows. From the defining equation, $S_{\boldsymbol{a}}^{-i j}$ contains the node $\left(-a_{\varsigma(1)} / a_{\varsigma(2)}, 0,0,-1\right)$ in $\left(X_{\boldsymbol{a}} \backslash T\right) \cap \mathbb{A}_{\varsigma}^{4}$. As in

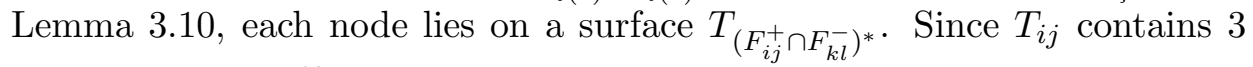
such surfaces, $S_{a}^{-i j}$ contains 3 nodes. This can be seen in Figure 7. 
Remark 3.17. Since $S_{\boldsymbol{a}}^{\varepsilon i j} \cap T \subset \overline{T_{F_{i j}^{\varepsilon *}}} \cap T=\varnothing$, the surfaces $S_{\boldsymbol{a}}^{\varepsilon i j}$ do not pass through any singularities $\boldsymbol{b} \in X_{\boldsymbol{a}} \cap T$, and may be ignored in considering resolutions of such singularities.

Definition 3.18. We define $\overline{X_{\boldsymbol{a}}}$ to be the blow up of $X_{\boldsymbol{a}}$ along all 10 surfaces $S_{\boldsymbol{a}}^{-i j}$. This is a projective small resolution of all 30 nodes in $X_{\boldsymbol{a}} \backslash T$.

Proposition 3.19. Let $\boldsymbol{a} \neq \phi(\boldsymbol{b})$ for any $\boldsymbol{b} \in \mathbb{P}^{4}$. Then the nodal Calabi-Yau variety $X_{\boldsymbol{a}}$ has a small projective resolution $\bar{X}_{\boldsymbol{a}}$.

Proof. This is because $X_{\boldsymbol{a}} \cap T$ is smooth in this case, by Lemma 3.7.

We have now constructed a five dimensional family of Calabi-Yau threefolds, $\bar{X}_{\boldsymbol{a}}$. In Proposition 4.8 we will see that $h^{12}$ of the general member is 5. By Proposition 3.2 the Euler number of $X_{\boldsymbol{a}}$ for general $\boldsymbol{a}$ is 80 , and so the Hodge diamond is as follows:

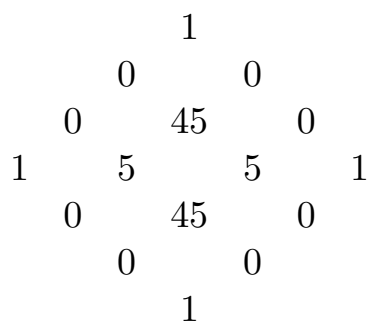

3.2.6. Big resolutions of singularities on $X_{\boldsymbol{a}} \cap T$

As discussed in Section 3.2.4, the big resolution of a singularity $\boldsymbol{b} \in$ $X_{\boldsymbol{a}} \cap T$ replaces $\boldsymbol{b}$ by a quadric, which in this case has equation given by the quadratic part of (15). By transforming (16) to a diagonal matrix, one can see that the rulings of this quadric are defined over the field

$$
\mathbb{Q}\left[\sqrt{-b_{0} b_{1} b_{2}\left(b_{0}+b_{1}+b_{2}\right)}, \sqrt{-b_{3} b_{4} b_{5}\left(b_{3}+b_{4}+b_{5}\right)}\right],
$$

and other similarly defined fields. For later use, we now consider the number of points on these quadrics over finite fields. First we need the following:

LEMMA 3.20. Let $f$ be an irreducible quadric in $\mathbb{P}^{3}\left(\mathbb{F}_{p}\right)$, for $p \neq 2$, with corresponding matrix $M \in M_{4}\left(\mathbb{F}_{p}\right)$. Then we have

$$
\#(f=0)\left(\mathbb{F}_{p}\right)= \begin{cases}(p+1)^{2} & \text { if } \operatorname{det} M \text { is a square in } \mathbb{F}_{p} \\ p^{2}+1 & \text { if } \operatorname{det} M \text { is not a square in } \mathbb{F}_{p} .\end{cases}
$$


Proof. This follows from [Se1, Proposition 5, IV §1.7].

Together with Proposition 3.8, this immediately implies the following:

Corollary 3.21. For the quadric $Q_{\boldsymbol{b}}$ introduced in resolving the singularity at $\boldsymbol{b}:=\left(b_{1}: b_{2}: b_{3}: b_{4}: b_{5}\right)$ on $X_{\phi(\boldsymbol{b})}$, we have

$$
\# Q_{\boldsymbol{b}}\left(\mathbb{F}_{p}\right)= \begin{cases}(p+1)^{2} & \text { if } \sqrt{b_{0} b_{1} b_{2} b_{3} b_{4} b_{5}} \in \mathbb{F}_{p} \\ p^{2}+1 & \text { if } \sqrt{b_{0} b_{1} b_{2} b_{3} b_{4} b_{5}} \notin \mathbb{F}_{p},\end{cases}
$$

where $b_{0}=b_{1}+b_{2}+b_{3}+b_{4}+b_{5}$.

3.2.7. Small resolutions of singularities on $X_{\boldsymbol{a}} \cap T$

Proposition 3.22. For $\boldsymbol{b}=(a: b:-b: c:-c$ ) (up to permutation of coordinates), there is a small projective resolution of $\boldsymbol{b} \in X_{\phi(\boldsymbol{b})}$.

Proof. In this case the surface $\left(X_{2}+X_{3}=X_{4}+X_{5}=0\right)$ lies in $X_{\phi(\boldsymbol{b})}$, contains the point $\boldsymbol{b}$, and its closure is a smooth Weil divisor in $X_{\phi(\boldsymbol{b})}$. Thus a small resolution is achieved by blowing up this surface.

Remark 3.23. Note that for $\boldsymbol{b}$ as in the proposition, $X_{\phi(\boldsymbol{b})}$ has at least four singularities, at $(a: b:-b: c:-c),(a:-b: b: c:-c),(a:-b: b:$ $-c: c)$ and $(a: b:-b:-c: c)$.

COROLlary 3.24. There is a small projective resolution of all the singularities of $X_{1}$.

Proof. This follows from Proposition 3.22, since if $\boldsymbol{a}=(1: 1: 1$ : $1: 1: 1)=\phi(\boldsymbol{b})$ then $\boldsymbol{b}=(1: 1: 1:-1:-1)$, up to permutation of coordinates.

Remark 3.25. The only Calabi-Yau threefolds in Table 1 for which all singularities can be resolved by the small resolution of Proposition 3.22 are $X_{\phi(\boldsymbol{b})}$ for $\boldsymbol{b}=(1:-1: b:-b: c)$ up to permutation, with $1 \pm b \pm c \neq 0$ for all sign choices; this can easily be seen by listing all possible singularities, by using (17) and (18). In Corollary 4.5 we will see that other $X_{\boldsymbol{a}}$ have no small projective resolution. 


\section{$\S 4 . \quad X_{a}$ as a fibre product}

We now show that $X_{\boldsymbol{a}}$ is birational to the fibre product of families of elliptic curves. This enables us to apply Schoen's results [Sc] on such threefolds.

First we define the families of elliptic curves.

Definition 4.1. For $a, b, c \in k \backslash\{0\}$ define an elliptic surface $\mathcal{E}_{a, b, c}$ to be the resolution of the surface $\mathcal{E}_{a, b, c}^{\prime} \subset \mathbb{P}^{2} \times \mathbb{P}^{1}$ given by

$$
\mathcal{E}_{a, b, c}^{\prime}:(x+y+z)(a x y+b y z+c z x) t_{0}=t_{1} x y z,
$$

where $(x: y: z) \in \mathbb{P}^{2},\left(t_{0}: t_{1}\right) \in \mathbb{P}^{1}$. There is a projection $p: \mathcal{E}_{a, b, c} \rightarrow \mathbb{P}^{1}$, with fibre $\mathcal{E}_{a, b, c, t}:=p^{-1}(1: t)$. We write $\mathcal{E}_{a}:=\mathcal{E}_{1,1, a}$ and $\mathcal{E}_{a, t}:=\mathcal{E}_{1,1, a, t}$.

The only singular points of $\mathcal{E}_{a, b, c}^{\prime}$ are the three singular points of the fibre at infinity. When these are resolved the fibre at infinity becomes an $I_{6}$ fibre.

When $\mathcal{E}_{a, b, c, t}$ is smooth, taking the zero to be $(0: 1:-1)$, the elliptic curve $\mathcal{E}_{a, b, c, t}$ is isomorphic to the cubic curve with equation

$$
y^{2}=x\left(\left(x+\frac{2\left(t^{2}+a^{2}+b^{2}+c^{2}\right)-(t+a+b+c)^{2}}{8 a^{2}}\right)^{2}-\frac{A(a, b, c, t)}{64 a^{4}}\right)
$$

where

$$
A(a, b, c, t):=\prod_{(\nu, \mu) \in\{-1,1\}^{2}}\left(t-(\sqrt{a}+\nu \sqrt{b}+\mu \sqrt{c})^{2}\right) .
$$

From this we find that the $j$-invariant of $\mathcal{E}_{a, b, c, t}$ is given by

$$
j\left(\mathcal{E}_{a, b, c, t}\right)=\frac{(A(a, b, c, t)+16 a b c t)^{3}}{(a b c t)^{2} A(a, b, c, t)} .
$$

This implies that in general $\mathcal{E}_{a, b, c}$ has 6 singular fibres, corresponding to the values of $t$ for which $j\left(\mathcal{E}_{a, b, c, t}\right)=\infty$. The singular fibres together with their fibre type, in the general and all special cases are given in Table 2. As examples, we also tabulate the data for $\mathcal{E}_{1}, \mathcal{E}_{9}, \mathcal{E}_{25}$ and $\mathcal{E}_{1,4,4}$.

LEMMA 4.2. $X_{\left(a_{1}: a_{2}: a_{3}: a_{4}: a_{5}: a_{6}\right)}$ and $\mathcal{E}_{a_{1}, a_{2}, a_{3}} \times_{\mathbb{P}^{1}} \mathcal{E}_{a_{4}, a_{5}, a_{6}}$ are birational. 
Proof. We can rewrite (12) as

$$
\begin{aligned}
X_{1}+X_{2}+X_{3} & =-\left(X_{4}+X_{5}+X_{6}\right), \\
\frac{a_{1}}{X_{1}}+\frac{a_{2}}{X_{2}}+\frac{a_{3}}{X_{3}} & =-\left(\frac{a_{4}}{X_{4}}+\frac{a_{5}}{X_{5}}+\frac{a_{6}}{X_{6}}\right),
\end{aligned}
$$

from which, introducing a new parameter $\left(\lambda_{0}: \lambda_{1}\right) \in \mathbb{P}^{1}$, we obtain

$$
\begin{aligned}
& \left(X_{1}+X_{2}+X_{3}\right)\left(\frac{a_{1}}{X_{1}}+\frac{a_{2}}{X_{2}}+\frac{a_{3}}{X_{3}}\right) \lambda_{0}=\lambda_{1}, \\
& \left(X_{4}+X_{5}+X_{6}\right)\left(\frac{a_{4}}{X_{4}}+\frac{a_{5}}{X_{5}}+\frac{a_{6}}{X_{6}}\right) \lambda_{0}=\lambda_{1},
\end{aligned}
$$

But these are equations for $\mathcal{E}_{a_{1}, a_{2}, a_{3}}$ and $\mathcal{E}_{a_{4}, a_{5}, a_{6}}$, so we have a map

$$
\begin{gathered}
\varphi_{T}: X_{\boldsymbol{a}} \cap T \longrightarrow\left(\mathcal{E}_{a_{1}, a_{2}, a_{3}} \cap\left(\lambda_{0} \lambda_{1} \neq 0\right)\right) \times_{\mathbb{P}^{1}}\left(\mathcal{E}_{a_{4}, a_{5}, a_{6}} \cap\left(\lambda_{0} \lambda_{1} \neq 0\right)\right), \\
\left(X_{1}: X_{2}: X_{3}: X_{4}: X_{5}: X_{6}\right) \longmapsto\left(X_{1}: X_{2}: X_{3}\right) \times\left(X_{4}: X_{5}: X_{6}\right) .
\end{gathered}
$$

If $P:=\left(X_{1}: X_{2}: X_{3}\right) \in \mathcal{E}_{a_{1}, a_{2}, a_{3}} \cap\left(\lambda_{0} \lambda_{1} \neq 0\right)$ and $Q:=\left(X_{4}: X_{5}: X_{6}\right) \in$ $\mathcal{E}_{a_{4}, a_{5}, a_{6}} \cap\left(\lambda_{0} \lambda_{1} \neq 0\right)$, then $\left(X_{1}+X_{2}+X_{3}\right)+\mu\left(X_{4}+X_{5}+X_{6}\right)=0$ for some unique $\mu \neq 0$, and then $R:=\left(X_{1}: X_{2}: X_{3}: \mu X_{4}: \mu X_{5}: \mu X_{6}\right) \in X_{\boldsymbol{a}}$ and $\varphi(R)=(P, Q)$. Thus $\varphi_{T}$ defines a birational map.

For example, this result implies that $X_{1}, X_{9}, X_{(1: 1: 1: 1: 4: 4)}$ and $X_{(1: 1: 1: 4: 4: 9)}$ are birational to $\mathcal{E}_{1} \times_{\mathbb{P}^{1}} \mathcal{E}_{1}, \mathcal{E}_{1} \times_{\mathbb{P}^{1}} \mathcal{E}_{9}, \mathcal{E}_{1} \times_{\mathbb{P}^{1}} \mathcal{E}_{1,4,4}$ and $\mathcal{E}_{9} \times_{\mathbb{P}^{1}} \mathcal{E}_{1,4,4}$ respectively.

\subsection{Existence of smooth projective Calabi-Yau models}

Now we can apply results of Schoen [Sc] to determine whether $X_{\boldsymbol{a}}$ has a small projective resolution or not.

LEMMA 4.3. If $a, b, c, d, e, f \in k \backslash\{0\}$ with $a+b+c+d+e+f=0$ satisfy (i) $0 \notin\{ \pm a \pm b \pm c, \pm d \pm e \pm f\}$, (ii) $(a+b+c)^{2} \notin\left\{(a+b-c)^{2},(a-b+\right.$ $\left.c)^{2},(a-b-c)^{2}\right\}$, and (iii) $\left\{a^{2}, b^{2}, c^{2}\right\} \neq\left\{d^{2}, e^{2}, f^{2}\right\}$, then $\mathcal{E}_{a^{2}, b^{2}, c^{2}} \times_{\mathbb{P}^{1}} \mathcal{E}_{d^{2}, e^{2}, f^{2}}$ has no small projective resolution.

Proof. This follows from [Sc, Lemma 3.1 (iii)], as in this case $\mathcal{E}_{a, b, c}$ and $\mathcal{E}_{d, e, f}$ are not isogenous, and from Table 2 , both have singular fibres at $(a+b+c)^{2}$, with that for $\mathcal{E}_{a, b, c}$ being $I_{1}$ type. 


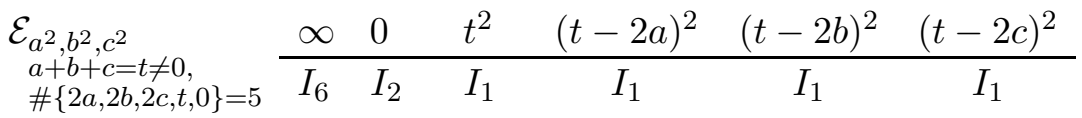

$$
\begin{aligned}
& \begin{array}{lccccc}
\mathcal{E}_{a^{2}, a^{2}, b^{2}} & \infty & 0 & b^{2} & (2 a-b)^{2} & (2 a+b)^{2} \\
\cline { 2 - 6 } \#\{b, a, 2 a, 0\}=4 & I_{6} & I_{2} & I_{2} & I_{1} & I_{1}
\end{array} \\
& \begin{array}{lllll}
\mathcal{E}_{\substack{a^{2}, a^{2}, a^{2} \\
a \neq 0}} & \infty & 0 & a^{2} & 9 a^{2} \\
\cline { 2 - 5 } & I_{6} & I_{2} & I_{3} & I_{1}
\end{array} \\
& \begin{array}{llllc}
\mathcal{E}_{a^{2}, a^{2}, 4 a^{2}} & \infty & 0 & 4 a^{2} & 16 a^{2} \\
\cline { 2 - 5 } a \neq 0 & I_{6} & I I I & I_{2} & I_{1}
\end{array}
\end{aligned}
$$

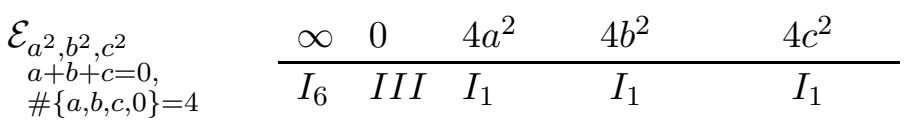

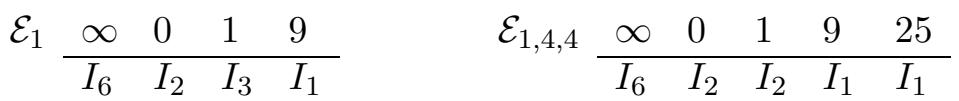

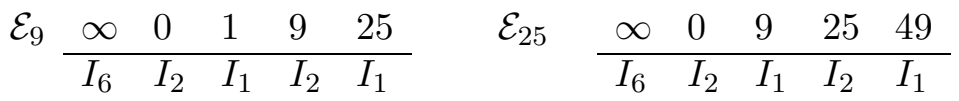

Table 2: The fibre types of the singular fibres of $\mathcal{E}_{\boldsymbol{a}}$.

In Corollary 3.24 we saw that $X_{1}$ has a smooth projective Calabi-Yau model. However, as we shall now see, this is not true for $X_{\phi(\boldsymbol{b})}$ in general. The following was pointed out to us by J. Kollár.

Proposition 4.4. Suppose $Y$ is a nodal threefold with trivial canonical bundle and that there is no small resolution of all singularities. Then $Y$ does not posess a smooth projective Calabi-Yau model.

Proof. Assume that $Z$ is a smooth projective model of $Y$ which is Calabi-Yau. We can successively blow up Weil divisors of $Y$ until we obtain a projective variety $Y^{\prime}$ with all Weil divisors of $Y^{\prime}$ Cartier at all nodes. In other words $Y^{\prime}$ is factorial. By assumption $Y^{\prime}$ cannot be smooth. We have a birational map $f: Y^{\prime} \rightarrow Z$. Since both $Y^{\prime}$ and $Z$ have trivial (and hence nef) canonical bundle the map $f$ factors as a finite sequence of flops [Ko, Theorem 4.9]. Since flops in dimension 3 do not change the type of singularity [Ko, Theorem 2.4] this gives a contradiction. 
Corollary 4.5. For $\boldsymbol{b} \in T$, the nodal variety $X_{\phi(\boldsymbol{b})}$ does not posess smooth projective Calabi-Yau model unless $\phi(\boldsymbol{b})=\phi((1:-1: b:-b: c))$, up to permutations, with $1 \pm b \pm c$ for some sign choice.

Proof. Let $\phi(\boldsymbol{b})=\left(b_{1}^{2}: b_{2}^{2}: b_{3}^{2}: b_{4}^{2}: b_{5}^{2}: b_{6}^{2}\right)$ with $b_{1}+b_{2}+b_{3}+$ $b_{4}+b_{5}+b_{6}=0$. If for some permutation of these variables the condition of Lemma 4.3 is satisfied, $\mathcal{E}_{b_{1}^{2}, b_{2}^{2}, b_{3}^{2}} \times_{\mathbb{P}_{1}^{1}} \mathcal{E}_{b_{4}^{2}, b_{5}^{2}, b_{6}^{2}}$ has no smooth projective CalabiYau model. Then Proposition 4.4 and Lemma 4.2 imply that $X_{\phi(\boldsymbol{b})}$ has no smooth projective Calabi-Yau model.

Suppose that conditions (i), (ii) and (iii) of Lemma 4.3 are not satisfied for any permutations, or sign changes preserving $\sum b_{i}=0$, of the $b_{i}$.

First note that $(a+b+c)^{2} \notin\left\{(a+b-c)^{2},(a-b+c)^{2},(a-b-c)^{2}\right\} \Longleftrightarrow$ $a b c(a+b)(b+c)(c+a) \neq 0$, so if $b_{i}+b_{j} \neq 0$ for $1 \leq i, j \leq 6$, condition (ii) can not fail. If we had $b_{i}+b_{j}+b_{k}=0$ for all triples $1 \leq i, j, k \leq 6$, then all $b_{i}$ are equal, but this contradicts $\sum b_{i}=0$. If (iii) fails for all permutations, then all $b_{i}^{2}$ are equal, and if $b_{i}+b_{j} \neq 0$, this again gives the contradiction that all $b_{i}$ are equal. Hence we can assume $b_{1}+b_{2}=0$. Now suppose that $b_{i}+b_{j} \neq 0$ for $3 \leq i, j \leq 6$. Then (ii) holds for $b_{3}, b_{4}, b_{5}$. Now $b_{3}+b_{4}+b_{5}+b_{6}=-\left(b_{1}+b_{2}\right)=0$, so (i) also holds, since $a_{6} \neq 0$. Similarly, (i) and (ii) hold for all other triples from $b_{3}, b_{4}, b_{5}, b_{6}$. If (iii) fails for all of these, then again, all $b_{i}^{2}$ are equal, but this is not possible with $\sum b_{i}=0$. Thus we can assume $b_{3}+b_{4}=0$, and since $\sum b_{i}=0$ we also have $b_{5}+b_{6}=0$.

Thus, if (i), (ii) or (iii) always fail, this implies that up to permutations, $\boldsymbol{b}=(1:-1: b:-b: c)$. If $1+ \pm b+ \pm c=0$, then after a sign change, we have $1+b+c=0$, and then $\phi(\boldsymbol{b})=(1: 1: b: b:-c)$, and taking $b_{i}$ in this order satisfies (i), (ii) and (iii) of Lemma 4.3 .

Conversely, if $\boldsymbol{b}=(1:-1: b:-b: c)$, then (i), (ii) or (iii) always fail, unless $\phi(\boldsymbol{b}) \neq \phi(\boldsymbol{c})$ for some $\boldsymbol{c}$ not of this form. As in Proposition 3.11, we obtain $\boldsymbol{c}$ from $\boldsymbol{b}$ by changing the signs of the $b_{i}$ in a nontrivial subset of the $b_{i}$ which sum to 0 . For $\boldsymbol{c}$ to have a different form is equivalent to $1 \pm b \pm c=0$ for some choice of signs.

In case $\phi(b)=\phi((1:-1: b:-b: c))$, up to permutations, with $1 \pm b \pm c \neq 0$ for all sign choices, by Proposition 3.22 and Remark 3.25 there is a small resolution of $X_{\phi(\boldsymbol{b})}$, by explicit construction.

In particular, this means that $X_{9}, X_{25}, X_{(1: 1: 1: 1: 4: 4)}$ and $X_{(1: 1: 1: 4: 4: 9)}$ have no small projective resolution. Later we will see that $X_{1}$ and $X_{9}$ have the same $L$-series. The Tate conjecture then says that there should be 
a correspondence between them. In this case such a correspondence can not be a birational map, since Corollaries 4.5 and 3.24 imply the following result.

Corollary 4.6. $X_{1}$ and $X_{9}$ are not birational.

\subsection{Computation of $h^{12}$}

By Proposition 3.2, we know all the Hodge numbers of the varieties $\widetilde{X}_{\boldsymbol{a}}$ and $\widehat{X}_{\boldsymbol{a}}$, except for $h^{11}$ and $h^{12}$, for which we only know the difference. Note that $\tilde{h}^{12}=\hat{h}^{12}$, so it is enough to compute $\tilde{h}^{12}$. We will use the fact that in our situation $h^{12}$ is a birational invariant. This can either be deduced from [Ko, Corollary (4.12)] or, as Batyrev has informed us, by using motivic integration.

Then from Lemma 4.2 it is enough to compute $h^{12}$ for the corresponding elliptic families fibre product. First we need to know that the elliptic families in question are semistable, for which we need the following lemma.

LEMmA 4.7. Suppose that $b_{1}, \ldots, b_{6} \neq 1,1,1,1,1,2$ or $1,1,1,1,2,3$ (up to scaling, permutations and sign changes). Then, after possible permutation, we can assume that $b_{1} \pm b_{2} \pm b_{3} \neq 0$ and $b_{4} \pm b_{5} \pm b_{6} \neq 0$, for all sign choices.

Proof. Suppose that this is not the case. Then for each partition $\left\{i_{1}, i_{2}, i_{3}\right\},\left\{i_{4}, i_{5}, i_{6}\right\}$ of $1, \ldots, 6$ into 2 sets of size 3 , we have either $\left(b_{i_{1}} \pm\right.$ $\left.b_{i_{2}} \pm b_{i_{3}}\right)=0$ or $\left(b_{i_{4}} \pm b_{i_{5}} \pm b_{i_{6}}\right)=0$, (or both) (for some choice of signs).

First we will show that some of the $b_{i}$ must be equal, up to sign. There are 10 partitions, so we have ten distinct sets $\left\{i_{1}, i_{2}, i_{3}\right\}$ of size 3 , with $\left(b_{i_{1}} \pm b_{i_{2}} \pm b_{i_{3}}\right)=0$. These sets have $30=3 \cdot 10$ entries, and so each of $1, \ldots, 6$ must occur at least $5=30 / 6$ times, i.e., in at least 5 of the 10 sets.

Take 5 of the sets containing 1 . Suppose we always have $b_{i} \neq \pm b_{j}$ for $i \neq j$. Then for each other element occurring in these 5 sets, it must occur exactly twice, with opposite signs; or else we would have, e.g., $b_{1}+\varepsilon b_{2} \pm b_{4}=$ 0 and $b_{1}+\varepsilon b_{2} \pm b_{5}=0$ where $\varepsilon= \pm 1$, so $b_{4}= \pm b_{5}$. But then when we take the sum of all 5 equalities, we would get $5 b_{1}=0$, a contradiction. Hence we have $b_{i}= \pm b_{j}$ for some $i, j \in\{2,3,4,5,6\}$. This is similarly the case for every other subset of 5 elements. This implies that there are two (not necessarily disjoint) pairs of equal elements amongst the $b_{i}$. Thus the $b_{i}$ have the form $a, a, a, b, c, d$ or $a, a, b, b, c, d$, up to sign. In the first case we 
must have $b \pm c \pm d=0$. From the other possible partitions, we get (possibly after replacing $b$ with $-b$ or $c$ with $-c$ ),

$$
\begin{array}{rll}
b= \pm 2 a & \text { or } & a+c+d=0 \\
\text { and } c= \pm 2 a & \text { or } & a+b \pm d=0, \\
\text { and } d= \pm 2 a & \text { or } & a \pm c \pm b=0 .
\end{array}
$$

We can not have all of the first column true, since this would imply $a=0$ by taking the sum (up to sign), since $b \pm c \pm d=0$. If two of the first column hold, e.g., $b= \pm 2 a$, and $c= \pm 2 a$, then from the last line we get $a= \pm 4 a$, a contradiction. If from the first column we just have $d= \pm 2 a$, then from the second, we get $b, c \in\{a,-3 a\}$; since $b \pm c \pm d=0$, we must have $b=c=a$ or $\{b, c\}=\{a,-3 a\}$. The first possibility gives that the $b_{i}$ are (after scaling) given, up to sign, by $1,1,1,1,2,3$, and the second gives $1,1,1,1,1,2$.

If the whole of the last column is true, then substituting $b= \pm c \pm d$ and eliminating $a$, we get $c+d= \pm c \pm 2 d$ or $\pm c$ and $c+d= \pm d \pm 2 c$ or $\pm d$. Since $c, d \neq 0$, this gives $2 c= \pm d$ or $\pm 3 d$ and $2 d= \pm c$ or $\pm 3 c$. But all possibilities lead to $c=d=0$, a contradiction.

Now suppose the $b_{i}$ have the form $a, a, b, b, c, d$. This gives us

$$
\begin{aligned}
& c= \pm 2 a \text { or } d= \pm 2 b, \\
& \text { and } d= \pm 2 a \text { or } c= \pm 2 b \text {. }
\end{aligned}
$$

If we have one true from each column, we get $b=a$, and this gives the previous case. Otherwise, we can assume we have $c= \pm d= \pm 2 a$. The $b_{i}$ now must have the form $a, a, b, b, 2 a, 2 a$. Thus $b=a$ or $b=2 a$. This gives, up to sign change, scaling and permutation, $1,1,1,1,2,2$ or $1,1,2,2,4,4$. But in the first case we have $(1 \pm 1 \pm 1)(1 \pm 2 \pm 2) \neq 0$, and in the second $(1 \pm 2 \pm 4)(1 \pm 2 \pm 4) \neq 0$, so neither of these is possible.

Hence the only possibility for the $b_{i}$ such that there is no permutation with $b_{1} \pm b_{2} \pm b_{3} \neq 0$ and $b_{4} \pm b_{5} \pm b_{6} \neq 0$, are those given.

Proposition 4.8. If $\mathcal{F}_{i}$ is the smallest set in Table 1 containing $\boldsymbol{a}$, then $h^{12}\left(\tilde{X}_{\boldsymbol{a}}\right)=\operatorname{dim} \mathcal{F}_{i}$. In particular, if $\boldsymbol{a} \in \mathbb{P}^{5} \backslash \phi\left(\mathbb{P}^{4}\right)$ with all coordinates nonzero, then $h^{12}\left(\bar{X}_{\boldsymbol{a}}\right)=5$.

Proof. For some $b_{i} \neq 0$ we have $\boldsymbol{a}=\left(b_{1}^{2}: \cdots: b_{6}^{2}\right) \in \mathbb{P}^{5}$. We set $\mathcal{E}^{1}=$

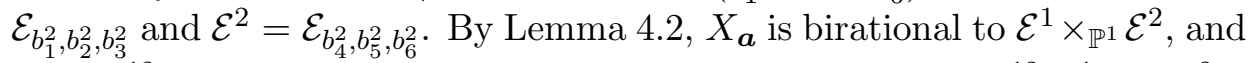
since $h^{12}$ is a birational invariant, it is enough to compute $h^{12}\left(\mathcal{E}^{1} \times_{\mathbb{P}^{1}} \mathcal{E}^{2}\right)$. 
Assuming $b_{i}$ are not $1,1,1,1,1,2$ or $1,1,1,1,2,3$, by Lemma 4.7 , we can assume that $b_{1} \pm b_{2} \pm b_{3}, b_{4} \pm b_{5} \pm b_{6} \neq 0$ for all sign choices, so $\mathcal{E}^{1}$ and $\mathcal{E}^{2}$ are semistable (see Table 2). Let $c_{i}(s)$ be the number of components of the fibre $\mathcal{E}_{s}^{i}, d=1$ if $\mathcal{E}^{1}$ and $\mathcal{E}^{2}$ are isogenous, and 0 otherwise, $S_{i}:=\{s \in$ $\mathbb{P}^{1} \mid \mathcal{E}_{s}^{i}$ is singular $\}$, and $S^{\prime}:=S_{1} \cap S_{2} \backslash\{0, \infty\}$. We have $S_{1}=\left\{\left(b_{1} \pm b_{2} \pm\right.\right.$ $\left.\left.b_{3}\right)^{2}\right\} \cup\{0, \infty\}, c_{1}(s)=\#\left\{\left(\varepsilon_{2}, \varepsilon_{3}\right) \in\{ \pm 1\}^{2} \mid\left(b_{1}+\varepsilon_{2} b_{2}+\varepsilon_{3} b_{3}\right)^{2}=s\right\}$, for $s \neq 0, \infty$, and $S_{2}$ and $c_{2}$ are given similarly in terms of $b_{4}, b_{5}, b_{6}$. Then by [Sc, (7.4)], for the smooth resolution $\mathcal{E}^{1 \times \times_{\mathbb{P}^{1}}} \mathcal{E}^{2}$ of $\mathcal{E}^{1} \times_{\mathbb{P}^{1}} \mathcal{E}^{2}$ we have

$$
\begin{aligned}
h^{12}\left(\widetilde{\mathcal{E}^{1} \times_{\mathbb{P}^{1}}} \mathcal{E}^{2}\right) & =\#\left(S_{1} \cup S_{2}\right)+d-5+\sum_{s \in \mathbb{P}^{1} \backslash\left(S_{1} \cap S_{2}\right)}\left(c_{1}(s) c_{2}(s)-1\right) \\
& =5+d-\# S^{\prime}-\sum_{s \in S^{\prime}}\left(c_{1}(s)+c_{2}(s)-2\right) \\
& =5+d-\sum_{s \in S^{\prime}}\left(c_{1}(s)+c_{2}(s)-1\right) \\
& =5+d-\sum_{s \in S^{\prime}} c_{1}(s) c_{2}(s)-\left(c_{1}(s)-1\right)\left(c_{2}(s)-1\right) .
\end{aligned}
$$

If $\left(b_{1}^{2}: \cdots: b_{6}^{2}\right) \in \mathcal{F}_{0}$, i.e., if $\sum \pm b_{i} \neq 0$ for all sign choices, then $d=0$ and $S^{\prime}=\varnothing$, so $h^{12}\left(\bar{X}_{\boldsymbol{a}}\right)=h^{12}\left(\widehat{\mathcal{E}^{1} \times \mathcal{E}^{2}}\right)=5$, as claimed.

Now suppose $\sum b_{i}=0$. Note we can take $d=0$, unless all the $\left|b_{i}\right|$ are all equal, since $d=1$ only if $\left|b_{i}\right|$ have the form $a, b, c, a, b, c$, but these can be rearranged as $a, a, b, c, c, b$ with $b \neq c, a$, unless all are equal. Set $A:=\sum_{s \in S^{\prime}}\left(c_{1}(s)-1\right)\left(c_{2}(s)-1\right)$. This is 0 unless the $\left|b_{i}\right|$ have the form $a, a, b, c, c, b$, with $b \neq a, c$ or $a, a, a, a, b, b$, with $b \neq a$, or $a, a, a, a, a, a$, in which case $A=1,2$ or 4 respectively. Now $-h^{12}+5+d+A$ is equal to

$$
\begin{aligned}
\# & \left\{\left(\varepsilon_{2}, \varepsilon_{3}, \varepsilon_{5}, \varepsilon_{6}\right) \in\{ \pm 1\}^{4} \mid\left(b_{1}+\varepsilon_{2} b_{2}+\varepsilon_{3} b_{3}\right)^{2}=\left(b_{4}+\varepsilon_{5} b_{5}+\varepsilon_{6} b_{6}\right)^{2}\right\} \\
& =\#\left\{\left(\varepsilon_{2}, \ldots, \varepsilon_{6}\right) \in\{ \pm 1\}^{5} \mid b_{1}+\varepsilon_{2} b_{2}+\varepsilon_{3} b_{3}+\varepsilon_{4} b_{4}+\varepsilon_{5} b_{5}+\varepsilon_{6} b_{6}=0\right\} \\
& =\text { number of singularities on } \bar{X}_{\boldsymbol{a}} .
\end{aligned}
$$

The first equality follows since if $u=b_{1}+\varepsilon_{2} b_{2}+\varepsilon_{3} b_{3}, v=b_{4}+\varepsilon_{5} b_{5}+\varepsilon_{6} b_{6}$, and $u^{2}=v^{2}$, then exactly one of $u= \pm v$ holds, since we assume $b_{1} \pm b_{2} \pm b_{3}$, 
$b_{4} \pm b_{5} \pm b_{6} \neq 0$. Proposition 3.11 gives the second equality. Now we have $h^{12}=5-$ number of singularities of $\bar{X}_{\boldsymbol{a}}+d+A$

$$
=5-\#\left(\operatorname{sings} \text { of } \bar{X}_{\boldsymbol{a}}\right)+ \begin{cases}0+1 & \text { if } \boldsymbol{a}=(1,1, b, c, c, b), b \neq 1, c, \\ 0+2 & \text { if } \boldsymbol{a}=(1,1,1,1, b, b), b \neq 1, \\ 1+4 & \text { if } \boldsymbol{a}=(1,1,1,1,1,1), \\ 0 & \text { otherwise. }\end{cases}
$$

The four cases correspond to $(1) \mathcal{F}_{6}$ and $\mathcal{F}_{10},(2) \mathcal{F}_{11}$ and $\mathcal{F}_{14},(3) \mathcal{F}_{15}$, and (4) everything else, respectively. Comparing the number of singularities with the dimension of $\mathcal{F}_{i}$ in Table 1 gives the result.

If the $b_{i}$ are $1,1,1,1,1,2$ or $1,1,1,1,2,3$, then instead of the above, we can use van Geemen's point counting method [GW] to show that the values of $h^{12}$ in these cases are also given as stated.

We immediately have the following, which also can be quickly deduced from [Sc, Proposition 7.1].

Corollary 4.9 . The varieties $X_{1}, X_{9}, X_{(1: 1: 1: 1: 4: 4)}$ and $X_{(1: 1: 1: 4: 4: 9)}$ are rigid, i.e., have $h^{12}=0$.

\section{§5. Elliptic surfaces in $X_{a}$}

Definition 5.1. Let $\boldsymbol{a}=\left(a_{1}: \cdots: a_{6}\right) \in \mathbb{P}^{5}, 1 \leq i<j \leq 5, i \neq j$, and $k<l<m$, with $\{i, j, k, l, m\}=\{1,2,3,4,5\}$. Suppose that

$$
\prod_{n=1}^{6} a_{n} \neq 0, a_{i}=a_{j}, \text { and } \sqrt{a}_{k} \pm \sqrt{a}_{l} \pm \sqrt{a}_{m} \pm \sqrt{a}_{6} \neq 0 .
$$

Let $H_{i j}$ denote the hyperplane given in $T$ by $X_{i}+X_{j}=0$. We define

$$
E_{\boldsymbol{a}}^{i j}:=\overline{\left(X_{\boldsymbol{a}} \cap H_{i j} \cap T\right)} \subset X_{\boldsymbol{a}},
$$

and let $\widetilde{E}_{\boldsymbol{a}}^{i j}$ be the strict transform of $E^{i j}$ in $\widetilde{\bar{X}}_{\boldsymbol{a}}$.

Substituting $X_{i}=-X_{j}$ in Equation (10) for $X_{\boldsymbol{a}} \cap T$, gives

$$
\left(X_{l}+X_{m}+X_{n}\right)\left(\frac{a_{l}}{X_{l}}+\frac{a_{m}}{X_{m}}+\frac{a_{n}}{X_{n}}\right)=a_{6} .
$$

This is the equation for $\mathcal{E}_{a_{l}, a_{m}, a_{n}, a_{6}}$ (see (20)), and so $E_{\boldsymbol{a}}^{i j}$ is birational to $\mathcal{E}_{a_{l}, a_{m}, a_{n}, a_{6}} \times \mathbb{P}^{1}$. 
Remark 5.2. For $\boldsymbol{a}$ satisfying condition (25), in terms of the fibre product structure, $X_{\boldsymbol{a}} \cong \mathcal{E}_{a_{i}, a_{i}, a_{6}} \times_{\mathbb{P}^{1}} \mathcal{E}_{a_{k}, a_{l}, a_{m}}, E_{\boldsymbol{a}}^{i j}$ corresponds to the component $L_{a_{6}} \times \mathcal{E}_{a_{k}, a_{l}, a_{m}, a_{6}}$ of the fibre over $a_{6}$, where $L_{a_{6}}$ is a component of the $I_{2}$ fibre $\mathcal{E}_{a_{i}, a_{i}, a_{6}, a_{6}}$ of the family $\mathcal{E}_{a_{i}, a_{i}, a_{6}}$.

Remark 5.3. With $\boldsymbol{a}$ as above, $\widetilde{E}_{\boldsymbol{a}}^{i j}$ is isomorphic to $\mathcal{E}_{a_{k}, a_{l}, a_{m}, a_{6}} \times \mathbb{P}^{1}$ blown up in the 6 points

$$
\begin{array}{lll}
(1: 0: 0) \times(1: 0), & (0: 1: 0) \times(1: 0), & (0: 0: 1) \times(1: 0), \\
(1:-1: 0) \times(0: 1), & (0: 1:-1) \times(0: 1), & (1: 0:-1) \times(0: 1) .
\end{array}
$$

Lemma 5.4. Let $a_{i}, a_{j}, a_{k}, a_{l}, a_{m}$ be as in Definition 5.1. Then $E_{\boldsymbol{a}}^{i j}$ is smooth and contains no singularities of $X_{\boldsymbol{a}} \cap T$.

Proof. Smoothness follows from considering all possible local equations, and the fact that $\sqrt{a}_{k} \pm \sqrt{a}_{l} \pm \sqrt{a}_{m} \pm \sqrt{a}_{6} \neq 0$ for all possible sign choices, so $\mathcal{E}_{a_{k}, a_{l}, a_{m}, a_{6}}$ is smooth.

By Lemma 3.7, a singularity on $X_{\boldsymbol{a}}$ has the form $\boldsymbol{b}=\left(b_{1}: \cdots: b_{5}\right)$ with $b_{i}^{2}=a_{i}$, and $\left(\sum b_{i}\right)^{2}=a_{6}$. But if $\boldsymbol{b} \in E_{\boldsymbol{a}}^{i j}$, then $b_{i}+b_{j}=0$, which implies $b_{k}+b_{l}+b_{m} \pm \sqrt{a_{6}}=0$. However this contradicts condition (25).

Definition 5.5. With $\boldsymbol{a}, i, j, k, l, m$ as in Definition 5.1, and $\mathcal{E}_{a_{k}, a_{l}, a_{m}, a_{6}}$ as in Definition 4.1 , let $\phi_{i j}$ be the birational map defined where all coordinates are nonzero by

$$
\begin{aligned}
& \phi_{i j}: \mathcal{E}_{a_{k}, a_{l}, a_{m}, a_{6}} \times \mathbb{P}^{1}--X_{\boldsymbol{a}}, \\
&(x: y: z) \times(r: s) \longmapsto\left(X_{1}: X_{2}: X_{3}: X_{4}: X_{5}\right), \\
& \text { with }\left(X_{i}: X_{j}: X_{l}: X_{m}: X_{n}\right)=(r z:-r z: s x: s y: s z) .
\end{aligned}
$$

We want to consider the subspace of $H_{3}\left(\widetilde{\bar{X}}_{\boldsymbol{a}}, \mathbb{Z}\right)$, spanned by the images of the induced maps

$$
\phi_{i j}^{*}: H_{1}\left(\mathcal{E}_{a_{k}, a_{l}, a_{m}, a_{6}}, \mathbb{Z}\right) \times H_{2}\left(\mathbb{P}^{1}, \mathbb{Z}\right) \longrightarrow H_{3}\left(\tilde{\bar{X}}_{\boldsymbol{a}}, \mathbb{Z}\right)
$$

Definition 5.6. For $\boldsymbol{c}=\left(c_{1}, c_{2}, c_{3}, c_{4}\right), c_{i} \neq 0, \sum \pm c_{i} \neq 0$, fix $\alpha_{\boldsymbol{c}}, \beta_{\boldsymbol{c}}$ to be 1 -cycles, with classes $\left[\alpha_{\boldsymbol{c}}\right],\left[\beta_{\boldsymbol{c}}\right]$ spanning $H_{1}\left(\mathcal{E}_{\boldsymbol{c}}, \mathbb{Z}\right)$, with $\alpha_{\boldsymbol{c}} \cdot \beta_{\boldsymbol{c}}=1$, and with

$(1: 0: 0),(0: 1: 0),(0: 0: 1),(1:-1: 0),(0: 1:-1),(1: 0:-1) \notin \alpha_{\boldsymbol{c}}, \beta_{\boldsymbol{c}}$. 
Definition 5.7. For $\boldsymbol{a}$ satisfying condition (25), and $\boldsymbol{c}=\left(a_{k}, a_{l}, a_{m}\right.$, $a_{6}$ ), define 3 -cycles on $X_{\boldsymbol{a}}$ by

$$
\alpha^{i j}:=\phi_{i j}\left(\alpha_{\boldsymbol{c}} \times \mathbb{P}^{1}\right), \quad \beta^{i j}:=\phi_{i j}\left(\beta_{\boldsymbol{c}} \times \mathbb{P}^{1}\right) .
$$

LEMMA 5.8. For a satisfying condition (25), we have

$$
A:=\left(\begin{array}{cc}
\left(\alpha^{i j}\right)^{2} & \alpha^{i j} \cdot \beta^{i j} \\
\beta^{i j} . \alpha^{i j} & \left(\beta^{i j}\right)^{2}
\end{array}\right)=\left(\begin{array}{rr}
0 & -2 \\
2 & 0
\end{array}\right) .
$$

Proof. We apply a general result in intersection theory (see [F, 19.2.2]). Namely, for a closed embedding $i: Y \hookrightarrow X$ of compact oriented manifolds, and $\alpha \in H_{r}(Y), \beta \in H_{s}(Y)$, we have

$$
i_{*}(\alpha) \cap i_{*}(\beta)=i_{*}\left(\left.Y\right|_{Y} \cap \alpha \cap \beta\right) .
$$

We take $Y=\widetilde{E}_{\boldsymbol{a}}^{i j} \sim_{\text {bir }} \mathcal{E} \times \mathbb{P}^{1}$, where $\mathcal{E}=\mathcal{E}_{a_{k}, a_{l}, a_{m}, a_{n}}$ for appropriate indices, and $X=\widetilde{\bar{X}}_{\boldsymbol{a}}$. We have

$$
\left.Y\right|_{Y}=\left.\left(K_{X}+Y\right)\right|_{Y}=K_{Y}=-2(\mathcal{E} \times\{\text { point }\})+\sum E_{j},
$$

where $E_{j}$ are the exceptional divisors in the blow up $\widetilde{E}_{\boldsymbol{a}}^{i j} \rightarrow \mathcal{E} \times \mathbb{P}^{1}$. The first equality of (27) follows from the fact that $K_{X}=\sum Q_{i}$, where the $Q_{i}$ are quadrics coming from blowing up nodes in $X_{\boldsymbol{a}} \cap T$; by Lemma 5.4 their restriction to $\widetilde{E}_{\boldsymbol{a}}^{i j}$ is trivial. The second equality comes from the adjunction formula. The third equality comes from the fact that the canonical bundle of the ruled surface $\mathcal{E} \times \mathbb{P}^{1}$ has degree -2 on the general fibre. Since $\alpha^{i j}$, $\beta^{i j}$ are chosen to avoid the $E_{j}$ (Remark 5.3 and Definition 5.6), we have

$$
\alpha^{i j} \cdot \beta^{i j}=\left.\alpha^{i j} \cap \beta^{i j} \cap Y\right|_{Y}=-2\left(\{\text { point }\} \times \mathbb{P}^{1}\right) \cap(\mathcal{E} \times\{\text { point }\})=-2 .
$$

The claim $\left(\alpha^{i j}\right)^{2}=\left(\beta^{i j}\right)^{2}=0$ can be seen directly from the geometry.

LEMMA 5.9. For $\boldsymbol{a}=\left(a_{1}: \cdots: a_{6}\right)$ with $a_{i}=a_{j}, a_{k}=a_{l}$, and $\pm a_{m} \pm a_{6} \notin\left\{0,2 \sqrt{a}_{i}, 2 \sqrt{a}_{k}\right\}$, where $\{i, j, k, l, m\}=\{1,2,3,4,5\}$, we have

$$
\left(\begin{array}{ll}
\alpha^{i j} \cdot \alpha^{k l} & \alpha^{i j} \cdot \beta^{k l} \\
\beta^{i j} \cdot \alpha^{k l} & \beta^{i j} \cdot \beta^{k l}
\end{array}\right)=0 .
$$

Proof. First note that substituting $X_{i}+X_{j}=X_{k}+X_{l}=0$ in (10) gives $a_{m}=a_{6}$, but we have assumed $\sqrt{a}_{m} \pm \sqrt{a}_{6} \neq 0$, so $H_{i j} \cap H_{l m} \cap X_{\boldsymbol{a}} \cap T=\varnothing$. Local considerations show that having picked $\alpha_{\boldsymbol{c}}$ and $\beta_{\boldsymbol{c}}$ to avoid certain points means that these cycles can also not intersect in $X_{\boldsymbol{a}} \backslash T$. 
LEMMA 5.10. For $\boldsymbol{a}=\left(a_{1}: \cdots: a_{6}\right)$ with $a_{i}=a_{j}=a_{k}$ and $\pm \sqrt{a_{l}} \pm$ $\sqrt{a_{m}} \pm \sqrt{a_{6}} \notin\left\{\sqrt{a}_{i}, 3 \sqrt{a}_{i}\right\}, i<j<k$ and $\{i, j, k, l, m\}=\{1,2,3,4,5\}$, we have

$$
\left(\begin{array}{ll}
\alpha^{i j} \cdot \alpha^{i k} & \alpha^{i j} \cdot \beta^{i k} \\
\beta^{i j} \cdot \alpha^{i k} & \beta^{i j} \cdot \beta^{i k}
\end{array}\right)=\operatorname{sg}\left(\phi_{i j}^{-1} \circ \phi_{i k}\right) B, \quad \text { where } B:=\left(\begin{array}{rr}
0 & 1 \\
-1 & 0
\end{array}\right),
$$

and where $\mathrm{sg}$ is the sign of $\phi_{i j}^{-1} \circ \phi_{i k}$ as a permutation of coordinates of $\mathbb{P}^{2}$.

Proof. By local considerations, one can show that the cycles do not meet in $X_{\boldsymbol{a}} \backslash T$. In $T$, the surfaces $E_{\boldsymbol{a}}^{i j}$ and $E_{\boldsymbol{a}}^{i k}$ meet in an elliptic curve, with $\alpha^{i j}$ and $\beta^{i j}$ restricting to the images of $\alpha$ and $\beta$ on this curve, so up to sign the intersection matrix is $B$ as above.

The sign is determined by whether or not the map $\phi_{k m}^{-1} \circ \phi_{i j}$ preserves the orientation of the chosen cycles. Suppose $i, j, k=1,2,4$. We have maps

$$
\begin{aligned}
& \phi_{12}:(x: y: z) \times(r: s) \longmapsto(r z:-r z: s x: s y: s z), \\
& \phi_{14}:(x: y: z) \times(r: s) \longmapsto(r z: s x: s y:-r z: s z) .
\end{aligned}
$$

Since $E_{\boldsymbol{a}}^{12} \cap E_{\boldsymbol{a}}^{14}$ is given by setting $X_{2}=X_{4}$, the map $\phi_{12}^{-1} \circ \phi_{14}$ is given by

$$
(x: y: z) \longmapsto(y: x: z) .
$$

This is an odd permutation of the coordinates, and so $\alpha_{14} \cap E_{12}$ and $\beta_{14} \cap$ $E_{12}$ have the opposite orientation to $\alpha_{12} \cap E_{14}$ and $\beta_{12} \cap E_{14}$, and so the intersection matrix is $-B$. Similar considerations hold in general.

Corollary 5.11. (i) If $\boldsymbol{a}=(1: 1: 1: 1: 1: t)$, for $t \neq 0,1,9$, then the intersection matrix of $\alpha_{\boldsymbol{a}}^{i j}, \beta_{\boldsymbol{a}}^{i j}$ for $1 \leq i<j \leq 5$ is given by the block matrix

$$
\begin{array}{rrrrrrrrrrr} 
& E^{12} & E^{13} & E^{14} & E^{15} & E^{23} & E^{24} & E^{25} & E^{34} & E^{35} & E^{45} \\
E^{12} & A & +B & -B & +B & +B & -B & +B & 0 & 0 & 0 \\
E^{13} & +B & A & +B & -B & +B & 0 & 0 & -B & +B & 0 \\
E^{14} & -B & +B & A & +B & 0 & +B & 0 & -B & 0 & +B \\
E^{15} & +B & -B & +B & A & 0 & 0 & +B & 0 & -B & +B \\
E^{23} & +B & +B & 0 & 0 & A & +B & -B & +B & -B & 0 \\
E^{24} & -B & 0 & +B & 0 & +B & A & +B & +B & 0 & -B \\
E^{25} & +B & 0 & 0 & +B & -B & +B & A & 0 & +B & -B \\
E^{34} & 0 & -B & -B & 0 & +B & +B & 0 & A & +B & +B \\
E^{35} & 0 & +B & 0 & -B & -B & 0 & +B & +B & A & +B \\
E^{45} & 0 & 0 & +B & +B & 0 & -B & -B & +B & +B & A
\end{array}
$$


This matrix has rank 8.

(ii) If $\boldsymbol{a}=(1: 1: 1: t: t: t)$, for $t \neq 0,1$, then the intersection matrix of the 6 three cycles $\alpha_{\boldsymbol{a}}^{i j}, \beta_{\boldsymbol{a}}^{i j}$ for $1 \leq i<j \leq 3$ is given by

$$
\left(\begin{array}{lll}
A & B & B \\
B & A & B \\
B & B & A
\end{array}\right)
$$

This matrix has rank 4.

Proof. This follows from Lemmas 5.8, 5.9 and 5.10. The conditions on $t$ in (i) and (ii) ensure that $1 \pm 1 \pm 1 \pm \sqrt{t}$ and $1 \pm \sqrt{t} \pm \sqrt{t} \pm \sqrt{t} \neq 0$, so that the elliptic curves $E_{\boldsymbol{a}}^{i j}$ are nonsingular.

We now look at how much of $H^{3}\left(\widetilde{\bar{X}}_{\boldsymbol{a}}\right)$ comes from the elliptic surfaces $E_{\boldsymbol{a}}^{i j}$. The maps $\phi_{i j}$, defined when $a_{i}=a_{j}$, and when the roots of the remaining coefficients can not sum to zero, gives us a homomorphism

$$
H_{\text {ét }}^{3}\left(\tilde{\bar{X}}_{\boldsymbol{a}}, \mathbb{Q}_{\ell}\right) \longrightarrow \bigoplus_{\substack{i, j \text { with } a_{i}=a_{j} \text { and } \\ \sum_{n \neq i, j} \pm a_{n} \neq 0}} H^{1}\left(E_{\boldsymbol{a}}^{i j}, \mathbb{Q}_{\ell}\right) \times H^{2}\left(\mathbb{P}^{1}, \mathbb{Q}_{\ell}\right) .
$$

Let $W_{\boldsymbol{a}}$ be the image of this map. The dimension of $W_{\boldsymbol{a}}$ can be determined by computing the dimension of the corresponding intersection matrix, as in the examples in Corollary 5.11. Let $V_{\boldsymbol{a}}$ be the kernel of this map. We have a sequence

$$
0 \longrightarrow V_{\boldsymbol{a}} \longrightarrow H_{\mathrm{ét}}^{3}\left(\widetilde{\bar{X}}_{\boldsymbol{a}}, \mathbb{Q}_{\ell}\right) \longrightarrow W_{\boldsymbol{a}} \longrightarrow 0
$$

of $\operatorname{Gal}(\overline{\mathbb{Q}} / \mathbb{Q})$ representations. (Strictly speaking the $\operatorname{Gal}(\overline{\mathbb{Q}} / \mathbb{Q})$ representation lives on the dual spaces but by abuse of language we shall still refer to the cohomology groups as $\operatorname{Gal}(\overline{\mathbb{Q}} / \mathbb{Q})$-modules.) By basic linear algebra

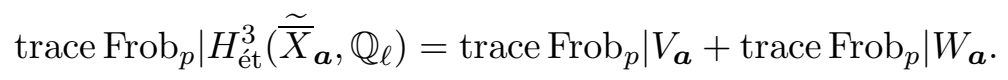

Since all elliptic curves over $\mathbb{Q}$ are modular, if $\boldsymbol{a} \in \mathbb{P}^{5}(\mathbb{Q})$, the Galois representation on $W_{\boldsymbol{a}}$ is given in terms of a weight 2 modular form, with level given by the conductor of the curve. This modular form can be determined by counting points. The values trace $\operatorname{Frob}_{p} \mid H_{\text {ét }}^{3}\left(\widetilde{\bar{X}}_{\boldsymbol{a}}, \mathbb{Q}_{\ell}\right)$ can also be determined by counting points, and so by subtraction we obtain the traces of the representation $V_{\boldsymbol{a}}$. We will be most interested in cases where $V_{\boldsymbol{a}}$ is 2-dimensional. These are the rigid examples of Corollary 4.9, and the following nonrigid cases. 
Corollary 5.12. For a given by one of the following,

$$
\begin{array}{ll}
\boldsymbol{a} & \operatorname{dim} \mathcal{F}_{i} \\
(1: 1: 1: 1: 1: 25) \in \mathcal{F}_{1} & 4 \\
(1: 1: 1: 9: 9: 9) \in \mathcal{F}_{4} & 2 \\
(1: 1: 4: 4: 4: 16) \in \mathcal{F}_{8} & 1
\end{array}
$$

The semi-simplification of the Galois representation on $H_{e t}^{3}\left(\widetilde{\bar{X}}_{\boldsymbol{a}}, \mathbb{Q}_{\ell}\right)$ splits into a sum of Galois representations corresponding to elliptic curves $W_{\boldsymbol{a}}$, and a 2-dimensional Galois-representation, $V_{\boldsymbol{a}}$.

Proof. The dimension of the pieces coming from elliptic surfaces $E_{\boldsymbol{a}}^{i j}$ for $1 \leq i \leq j \leq M$, with $M=5,3$ and 2 respectively, is given by Corollary 5.11, parts (i), (ii), and Lemma 5.8 respectively. These values of $\boldsymbol{a}$ are in the indicated families $\mathcal{F}_{i}$, defined in Table 1 , and in no smaller families. In each case we have that $\operatorname{dim} W_{\boldsymbol{a}}=h^{12}\left(\widetilde{\bar{X}}_{\boldsymbol{a}}\right)=\operatorname{dim} \mathcal{F}_{i}$, (see Proposition 4.8) and so Galois representation $V_{\boldsymbol{a}}$, which is the kernel, must have dimension 2.

Remark 5.13. In the next section we will compute the Galois representations of the $V_{\boldsymbol{a}}$ in the above corollary, and show that they correspond to weight 4 modular forms.

\section{§6. Computing the $L$-series}

We will use the Lefschetz fixed point theorem to compute coefficients of the $L$-series of $H_{\text {ét }}^{3}\left(\widetilde{\bar{X}}_{\boldsymbol{a}}\right)$. This says that for a variety $Z$ defined over $\mathbb{Q}$, the number of rational points of $Z$ over $\mathbb{F}_{p}$ is given by

$$
\# Z\left(\mathbb{F}_{p}\right)=\sum(-1)^{j}\left(\left.\operatorname{Frob}_{p}^{*}\right|_{H_{\text {ét }}^{j}(Z)}\right)
$$

The spaces $H_{\text {ét }}^{0}\left(\widetilde{\bar{X}}_{\boldsymbol{a}}\right)$ and $H_{\text {ét }}^{6}\left(\tilde{\bar{X}}_{\boldsymbol{a}}\right)$ are 1 dimensional, and Frob $_{p}$ acts trivially and by multiplication by $p^{3}$ respectively. The following result gives some information about the Galois action on $H_{\text {ét }}^{2}\left(\widetilde{\widetilde{X}}_{\boldsymbol{a}}\right)$, and (by duality) on $H_{\text {ét }}^{4}\left(\widetilde{\bar{X}}_{\boldsymbol{a}}\right)$.

Proposition 6.1. For a prime $p$ of good reduction for $\widetilde{\bar{X}}_{\boldsymbol{a}}$, all eigenvalues of the Frobenius action of Frob $_{p}$ on $H_{e ́ t}^{2}\left(\widetilde{X}_{\boldsymbol{a}}\right)$ are equal to $p$, provided the rulings of the quadrics $Q_{i}$ which are obtained by blowing up the 30 singularities are defined over the field $\mathbb{F}_{p}$. 
Proof. We claim that $H_{\text {ét }}^{2}(\widetilde{P}) \cong H_{\text {ét }}^{2}\left(X_{\boldsymbol{a}}\right)$, where $\widetilde{P}$ is the ambient toric variety. This suffices since $\widetilde{P}$ is a smooth toric variety and hence $H^{2}(\widetilde{P}, \mathbb{Z})$ is spanned by divisors defined over $\mathbb{Z}$. In order to prove that the restriction $H^{2}(\widetilde{P}, \mathbb{Q}) \rightarrow H^{2}\left(X_{\boldsymbol{a}}, \mathbb{Q}\right)$ is an isomorphism we proceed as follows. We first observe that as in the proof of $[\mathrm{C},(1.28)]$ one has an isomorphism $H^{2}\left(X_{\boldsymbol{a}}, \mathbb{Q}\right) \cong H^{2}\left(X_{u}, \mathbb{Q}\right)$ where $X_{u}$ is a general (smooth) Calabi-Yau in $\left|-K_{\widetilde{P}}\right|$. Using this isomorphism it is enough to show that $H^{2}(\widetilde{P}, \mathbb{Q}) \rightarrow$ $H^{2}\left(X_{u}, \mathbb{Q}\right)$ is an isomorphism. Both vector spaces have dimension 26. In the case of $X_{u}$ this was shown in Proposition 3.1, and for $\widetilde{P}$ this is standard toric geometry. (Viewing $\widetilde{P}$ as a repeated blow up of $\mathbb{P}^{4}$ one sees that the Picard group of $\widetilde{P}$ is spanned by the pullback of the hyperplane sections and the $5+10+10=25$ exceptional divisors. Note also that there are 5 $T$-invariant hyperplanes in $\widetilde{P}$ which are, of course, linearly equivalent). It is shown in the proof of [Ba, Theorem 4.42] that the Picard group of $X_{u}$ is spanned by components of divisors of the form $Y=H \cap X_{u}$ where $H$ is a $T$ invariant divisor on $\widetilde{P}$. In our case there are no proper faces of $\Delta$ which have interior points. Again from the proof of [Ba, Theorem 4.42, p. 520] one can conclude that $Y$ is always irreducible and hence $H^{2}(\widetilde{P}, \mathbb{Q}) \rightarrow H^{2}\left(X_{u}, \mathbb{Q}\right)$ is an epimorphism. Since both vector spaces have the same dimension this is indeed an isomorphism.

In order to go from $X_{\boldsymbol{a}}$ to $\widetilde{X}_{\boldsymbol{a}}$ we consider the Leray spectral sequence

$$
0 \longrightarrow H_{\text {êt }}^{2}\left(X_{\boldsymbol{a}}\right) \longrightarrow H_{\text {êt }}^{2}\left(\widetilde{X}_{\boldsymbol{a}}\right) \longrightarrow \bigoplus_{i=1}^{s} H_{\text {èt }}^{2}\left(Q_{i}\right)
$$

Hence it is enough to check that the rulings of the quadrics $Q_{i}$ are defined over $\mathbb{F}_{p}$.

We shall see later that to compute values of $\operatorname{Frob}_{p}$ acting on $H_{\text {ét }}^{3}\left(\widetilde{\bar{X}}_{\boldsymbol{a}}\right)$, it will be enough to count points on $X_{\boldsymbol{a}}$ over finite fields.

\subsection{Counting points on $\widetilde{X}_{a}$}

In this section we give a formula for counting points on $\widetilde{\bar{X}}_{\boldsymbol{a}}$ over finite fields. First we determine the primes of bad reduction for $X_{\boldsymbol{a}}$, since we will only count points on $X_{\boldsymbol{a}}$ at primes of good reduction.

Lemma 6.2. Let $\boldsymbol{a}=\left(a_{1}: \cdots: a_{6}\right) \in \mathbb{P}^{5}(\mathbb{Z})$, with $\prod_{i=1}^{5} a_{i} \neq 0$, and let $F(\boldsymbol{a})$ be the degree 16 polynomial in $\mathbb{Z}\left[a_{1}, \ldots, a_{6}\right]$ given by

$$
F(\boldsymbol{a})=\prod_{\left(\epsilon_{1}, \ldots, \epsilon_{5}\right) \in\{ \pm 1\}^{5}}\left(\sum_{i=1}^{5} \epsilon_{i} \sqrt{a}_{i}+\sqrt{a}_{6}\right) .
$$


If $\boldsymbol{a} \notin \phi\left(\mathbb{P}^{4}\right)$, then $\bar{X}_{\boldsymbol{a}} \otimes \mathbb{F}_{p}$ is smooth over $\overline{\mathbb{F}}_{p}$ for $p \nmid a_{1} a_{2} a_{3} a_{4} a_{5} a_{6} F(\boldsymbol{a})$.

Furthermore,

$\widetilde{X}_{1} \otimes \mathbb{F}_{p}, \widetilde{X}_{9} \otimes \mathbb{F}_{p}$ and $\widetilde{X}_{(1: 1: 1: 9: 9: 9)} \otimes \mathbb{F}_{p} \quad$ are smooth if $p \neq 2,3$,

$\widetilde{X}_{25} \otimes \mathbb{F}_{p}, \widetilde{X}_{(1: 1: 1: 1: 4: 4)} \otimes \mathbb{F}_{p}$ and $\widetilde{X}_{(1: 1: 4: 4: 4: 16)} \otimes \mathbb{F}_{p}$

are smooth if $p \neq 2,3,5$,

$\widetilde{X}_{(1: 1: 1: 4: 4: 9)} \otimes \mathbb{F}_{p}$ is smooth if $p \neq 2,3,5,7$.

Proof. Lemmas 3.10 and 3.7 describe the singularities of $X_{\boldsymbol{a}}$ over any field $\mathbb{F}_{p}$ with $p \nmid a_{i}$. The resolutions we have described over $\overline{\mathbb{Q}}$ remain resolutions over $\overline{\mathbb{F}}_{p}$. Thus if $\boldsymbol{a} \notin \phi\left(\mathbb{P}^{4}\right), \bar{X}_{\boldsymbol{a}} \otimes \mathbb{F}_{p}$ is smooth over $\overline{\mathbb{F}}_{p}$ unless $\boldsymbol{a} \equiv \phi(\boldsymbol{b}) \bmod p$ for some $\boldsymbol{b}$. This is the case only if $F(\boldsymbol{a}) \equiv 0 \bmod p$.

If $\boldsymbol{a}=\phi(\boldsymbol{b})$, the primes of bad reduction are the prime factors of $a_{i}$, and of the nonzero factors of $F(\boldsymbol{a})$. E.g., if all $a_{i}=1$, then $\left|\sum \pm \sqrt{a_{i}}\right|=0,2,4$ or 6 , so the primes of bad reduction are 2,3 . Other examples are computed similarly.

We now count points on $X_{\boldsymbol{a}}$ by considering points on $X_{\boldsymbol{a}} \cap T$ and on $X_{\boldsymbol{a}} \backslash T$, and points added in the resolution of singularities, separately.

LEMMA 6.3. If $\boldsymbol{a}=\left(a_{1}: a_{2}: a_{3}: a_{4}: a_{5}: a_{6}\right) \in \mathbb{P}^{5}\left(\mathbb{F}_{p}^{\times}\right)$, then

$$
\begin{aligned}
\#\left(X_{\boldsymbol{a}} \cap T\right)\left(\mathbb{F}_{p}\right) & \\
= & \sum_{y, z, w=1}^{p-1}\left(\left(\frac{\left((1+x+y+z)\left(\frac{a_{2}}{x}+\frac{a_{3}}{y}+\frac{a_{4}}{z}+a_{5}\right)-a_{1}-a_{6}\right)^{2}-4 a_{1} a_{6}}{p}\right)+1\right) \\
& \quad-2\left(p^{2}-3 p+3\right)+\rho\left(a_{1}, a_{6}\right)\left(\# \mathcal{E}_{a_{2}, a_{3}, a_{5}, a_{4}}\left(\mathbb{F}_{p}\right)-6\right),
\end{aligned}
$$

where $\rho\left(a_{1}, a_{6}\right)=p$ if $a_{1} \equiv a_{6} \bmod p$, and 0 otherwise, $\left(\frac{x}{p}\right)$ is the Kronecker symbol, and $\mathcal{E}_{a_{2}, a_{3}, a_{5}, a_{4}}\left(\mathbb{F}_{p}\right)$ is the elliptic curve given by (20).

Proof. We must compute the number of solutions to (10) with all $X_{i} \neq 0$. Setting $X_{5}=1, A=X_{2}+X_{3}+X_{4}+1$ and $B=a_{2} / X_{2}+a_{3} / X_{3}+$ $a_{4} / X_{4}+a_{5}$, and multiplying through by $X_{1}$, (10) becomes

$$
B X_{1}^{2}+\left(A B+a_{1}-a_{6}\right) X_{1}+a_{1} A=0 .
$$

For fixed $X_{2}, X_{3}, X_{4}$, this has $\left(\frac{d}{p}\right)+1$ solutions, where $d=\left(A B-a_{1}-\right.$ $\left.a_{6}\right)-4 a_{1} a_{6}$ is the discriminant of (33). This gives the term which is the sum in (32). However, 
(i) this sum counts solutions to (33) where $X_{1}=0$,

(ii) if $A=B=0, a_{1}=a_{6}$, there are $p-1$ solutions, but the sum counts 1 .

(iii) If $B=0, a_{1}=a_{6}, A \neq 0$, there are 0 solutions, but the sum counts 1 .

If $a_{1}=a_{6}$, then (i) occurs exactly when $A=0$ and $B \neq 0$. We must add

$$
\begin{aligned}
& -\#\left\{\left(X_{2}, X_{3}, X_{4}\right) \in\left(\mathbb{F}_{p}^{\times}\right)^{3} \mid A=0\right\} \\
& +\#\left\{\left(X_{2}, X_{3}, X_{4}\right) \in\left(\mathbb{F}_{p}^{\times}\right)^{3} \mid A=B=0\right\} p \\
& -\#\left\{\left(X_{2}, X_{3}, X_{4}\right) \in\left(\mathbb{F}_{p}^{\times}\right)^{3} \mid B=0\right\}
\end{aligned}
$$

to the sum in (32). Since $A=0, X_{2} X_{3} X_{4} \neq 0$ is a plane with 3 lines removed, the set in line $(34)$ has $p^{2}-3(p-1)$ points. The set in line (36) similarly has $p^{2}-3(p-1)$ points. The equations $A=B=0$ for the set in line (35) can be rearranged to give the the equation for $\mathcal{E}_{a_{2}, a_{3}, a_{5}, a_{4}}$, with all coordinates nonzero. Thus in the case $a_{1}=a_{6}$ we obtain (32).

The case $a_{1} \neq a_{6}$ is similar.

LEMMA 6.4. We have

$$
\#\left(X_{\boldsymbol{a}} \backslash T\right)\left(\mathbb{F}_{p}\right)=50 p^{2}+10 p+20 .
$$

Proof. The components of the decomposition $X_{\boldsymbol{a}}=\bigsqcup_{\sigma \in \widetilde{\Sigma}}\left(X_{\boldsymbol{a}} \cap T_{\sigma}\right)$ are listed in Table 3, and illustrated in Figure 7. If $\operatorname{dim} T_{\sigma}<4$, then $\widetilde{X}_{\boldsymbol{a}} \cap T_{\sigma}$ is rational, and so the number of points $\#\left(\widetilde{X}_{\boldsymbol{a}} \cap T_{\sigma}\right)\left(\mathbb{F}_{p}\right)$, can easily be computed, and is given in Table 4 . From Tables 3 and 4 we have

$$
\begin{aligned}
\#\left(X_{\boldsymbol{a}} \backslash T\right)\left(\mathbb{F}_{p}\right)=10 & \left(p^{2}-3 p+3\right)+20\left(2 p^{2}-6 p+5\right) \\
& +40(p-2)+60(p-1)+30(2 p-3)+120,
\end{aligned}
$$

which sums to give the required result.

LEMMA 6.5. For $\boldsymbol{a}=\left(a_{1}: \cdots: a_{6}\right) \in \mathbb{P}^{5}(\mathbb{Q})$, if the big resolution $\widetilde{\bar{X}}_{\boldsymbol{a}}$ of $\bar{X}_{\boldsymbol{a}}$ has smooth reduction $\bmod p$, then

$$
\begin{aligned}
& \# \widetilde{\bar{X}}_{\boldsymbol{a}}\left(\mathbb{F}_{p}\right)=48 p^{2}+46 p+14 \\
& +\sum_{x, y, z=1}^{p-1}\left(\left(\frac{\left((1+x+y+z)\left(\frac{a_{2}}{x}+\frac{a_{3}}{y}+\frac{a_{4}}{z}+a_{5}\right)-a_{1}-a_{6}\right)^{2}-4 a_{1} a_{6}}{p}\right)+1\right) \\
& +\sum_{\boldsymbol{b}=\left(b_{1}: \cdots: b_{5}\right) \in \mathbb{P}^{4}, \phi(\boldsymbol{b})=\boldsymbol{a}} p\left(p+1+\left(\frac{b_{1} b_{2} b_{3} b_{4} b_{5}\left(\sum b_{i}\right)}{p}\right)\right) \\
& +\rho\left(a_{1}, a_{6}\right)\left(\# \mathcal{E}_{a_{2}, a_{3}, a_{5}, a_{4}}\left(\mathbb{F}_{p}\right)-6\right),
\end{aligned}
$$




$$
\begin{aligned}
\widetilde{\bar{X}}_{\boldsymbol{a}}= & \text { The open threefold } \widetilde{X}_{\boldsymbol{a}} \cap T, \\
& +10 \text { translates of the surface } \widetilde{X}_{\boldsymbol{a}} \cap(x=0), \\
& +20 \text { translates of the surface } \widetilde{X}_{\boldsymbol{a}} \cap(y=0), \\
& +40 \text { translates of the curve } \widetilde{X}_{\boldsymbol{a}} \cap(x=y=0), \\
& +60 \text { translates of the curve } \widetilde{X}_{\boldsymbol{a}} \cap(x=z=0), \\
& +30 \text { translates of the curve } \widetilde{X}_{\boldsymbol{a}} \cap(y=z=0), \\
& +120 \text { translates of the point }(x, y, z, w)=(0,0,0,-1), \\
& +30 \mathbb{P}^{1} \text { s obtained in resolving the singularities in } X_{\boldsymbol{a}} \backslash T, \\
& + \text { the } Q_{\boldsymbol{b}} \text { obtained in the resolution of singularities in } X_{\boldsymbol{a}} \cap T .
\end{aligned}
$$

Table 3: Decomposition of $\widetilde{\bar{X}}_{\boldsymbol{a}}$. Translates mean images under the $S_{5}$ action.

$$
\begin{array}{llll} 
& \text { defining equations of } X_{\boldsymbol{a}} \cap T_{\sigma} & \#\left(X_{\boldsymbol{a}} \cap T_{\sigma}\right)\left(\mathbb{F}_{p}\right) \\
x=0 & y z w \neq 0 & a_{1}(1+w+w z+w z y)=0 & p^{2}-3 p+3 \\
y=0 & x z w \neq 0 & \left(a_{1}+a_{2} x\right)(1+w+w z)=0 & 2 p^{2}-6 p+5 \\
x=y=0 & z w \neq 0 & a_{1}(1+w+w z)=0 & p-2 \\
x=z=0 & y w \neq 0 & a_{1}(1+w)=0 & p-1 \\
y=z=0 & x w \neq 0 & \left(a_{1}+a_{2} x\right)(1+w)=0 & 2 p-3
\end{array}
$$

Table 4: Number of points on $X_{\boldsymbol{a}} \cap T_{\sigma}$.

where $\rho\left(a_{1}, a_{6}\right)=p$ if $a_{1} \equiv a_{6} \bmod p$, and 0 otherwise, $\left(\frac{x}{p}\right)$ is the Kronecker symbol, and $\mathcal{E}_{a_{2}, a_{3}, a_{5}, a_{4}}\left(\mathbb{F}_{p}\right)$ is the elliptic curve given by (20).

Proof. Table 3 lists the components of $\widetilde{\bar{X}}_{\boldsymbol{a}}$. By Proposition 3.10, if $\boldsymbol{a} \in \mathbb{P}^{5}(\mathbb{Z})$, all 30 nodes on $X_{\boldsymbol{a}} \backslash T$ are defined over $\mathbb{Q}$, and the $\mathbb{P}^{1}$ s added in the small resolution contribute $30 p$ to the sum. If $\boldsymbol{a}=\phi(\boldsymbol{b})$, Corollary 3.21 gives the number of points on the quadric $Q_{\boldsymbol{b}}$ introduced when $\boldsymbol{b}$ is blown up. Thus the number of points added in resolving the singularities of $X_{\boldsymbol{a}}$ is

$$
30 p+\sum_{\boldsymbol{b}=\left(b_{1}: \cdots: b_{5}\right) \in \mathbb{P}^{4}, \phi(\boldsymbol{b})=\boldsymbol{a}}\left(p+1+\left(\frac{b_{1} b_{2} b_{3} b_{4} b_{5} \sum_{i=1}^{5} b_{i}}{p}\right)\right) p .
$$

Adding up (32), (37) and (38) gives the result. 


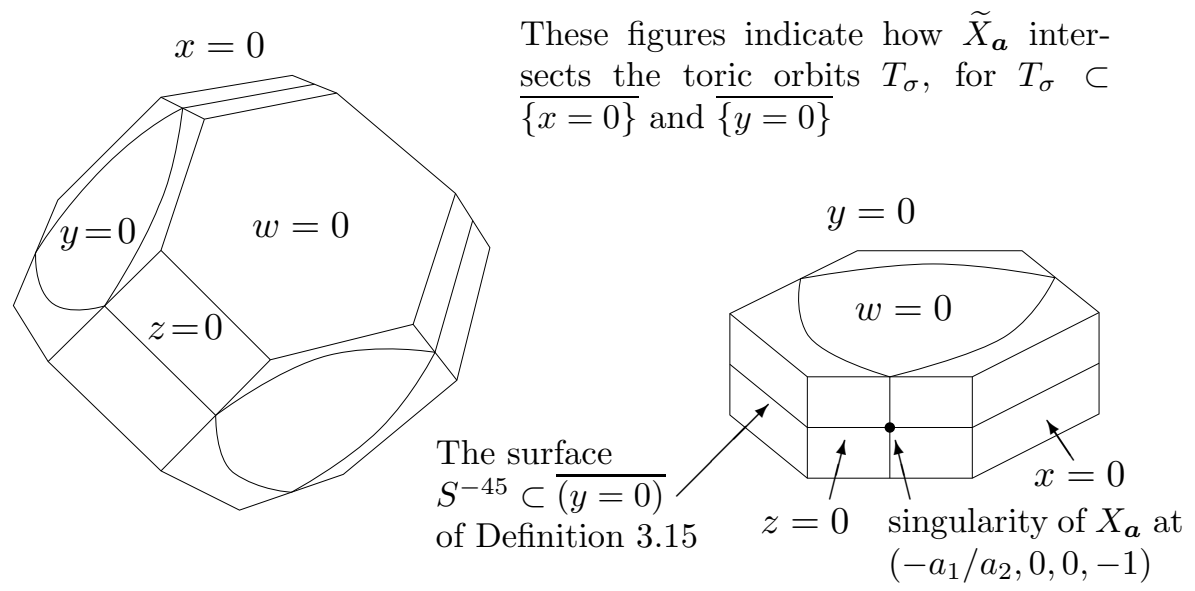

Figure 7: How $\widetilde{X}_{\boldsymbol{a}}$ intersects $P \backslash T$.

\subsection{Applying Livné's method}

We now want to prove that the 2 dimensional Galois representations $V_{\boldsymbol{a}}$, for $\boldsymbol{a}=(1: 1: 1: 1: 1: 1),(1: 1: 1: 1: 1: 9),(1: 1: 1: 1: 1: 25)$, $(1: 1: 1: 1: 4: 4),(1: 1: 1: 4: 4: 9),(1: 1: 4: 4: 4: 16)$ and (1:1:1:9:9:9) (see Corollaries 4.9, and 5.12) are modular, by comparing explicit computation and comparison of the coefficients of these $L$-series with coefficients of certain modular forms. In order to do this, we apply Falting's method, as given by Serre and Livné [Li, Theorem 4.3]. A simplified form is as follows:

Theorem 6.6. (Faltings-Serre-Livné) Let $\rho_{1}$ and $\rho_{2}$ be two 2-adic 2dimensional Galois representations, unramified outside a set of primes $S$. Let $K_{S}$ be the smallest field containing all quadratic extensions of $\mathbb{Q}$ ramified at primes in $S$, and let $T$ be a set of primes disjoint from $S$. Then if

(L.1) trace $\rho_{1} \equiv \operatorname{trace} \rho_{2} \equiv 0$ and $\operatorname{det} \rho_{1} \equiv \operatorname{det} \rho_{2}$,

(L.2) $\left\{\left.\operatorname{Frob}_{p}\right|_{K_{s}}: p \in T\right\}$ is "non-cubic" in $\mathrm{Gal}\left(K_{s} / K\right)$; in particular, it is sufficient for these sets to be equal,

(L.3) for all $p \in T$,

(a) trace $\rho_{1} \operatorname{Frob}_{p}=$ trace $\rho_{2} \operatorname{Frob}_{p}$,

(b) $\operatorname{det} \rho_{1} \operatorname{Frob}_{p}=\operatorname{det} \rho_{2} \operatorname{Frob}_{p}$,

then $\rho_{1}$ and $\rho_{2}$ have isomorphic semi-simplifications. 
We want to apply this result to the Galois representation on $H_{\text {ét }}^{3}\left(\widetilde{\bar{X}}_{\boldsymbol{a}} \otimes\right.$ $\left.\overline{\mathbb{F}}_{p}, \mathbb{Q}_{\ell}\right)$ and the modular Galois representation corresponding to a cuspidal Hecke eigenform, constructed by Serre and Deligne. Thus we need to do the following:

(L.1a) Check that trace $\left(\operatorname{Frob}_{p} \mid H_{\text {ét }}^{3}\left(\widetilde{\bar{X}}_{\boldsymbol{a}} \otimes \overline{\mathbb{F}}_{p}, \mathbb{Q}_{\ell}\right)\right)$ is always even for primes $p$ of good reduction.

(L.1b) Check that the coefficients $a_{p}$ of the modular forms in question are all even for primes $p$ not dividing the level.

(L.1c) and (L.3b) Remark that the determinants of both representations are given by $\chi^{3}$, where $\chi$ is the cyclotomic character.

(L.2) Determine a suitable set of primes $T_{s}$.

(L.3a) Compute trace $\left(\operatorname{Frob}_{p} \mid H_{\text {ét }}^{3}\left(\widetilde{\bar{X}}_{\boldsymbol{a}} \otimes \overline{\mathbb{F}}_{p}\right)\right)$ for all $p \in T_{s}$, and verify these are equal to the corresponding coefficients of the modular forms.

We now treat each of these points in turn.

(L.1a) We first remark that the trace of Frobenius on $H_{\text {ét }}^{2}\left(\widetilde{X}_{\boldsymbol{a}}\right)$ is an integer multiple of $p$ (and hence by duality the trace of Frobenius on $H_{\text {ét }}^{4}\left(\widetilde{X}_{\boldsymbol{a}}\right)$ is an integer multiple of $p^{2}$ ). This can be deduced either from the proof of Proposition 6.1 or directly from the analogue of the Riemann hypothesis. Thus the coefficients of the $L$-series are given by

$$
\operatorname{trace}\left(\operatorname{Frob}_{p} \mid H_{\text {ét }}^{3}\left(\widetilde{\bar{X}}_{\boldsymbol{a}} \otimes \overline{\mathbb{F}}_{p}\right)\right)=p^{3}+p(p+1) h+1-\# \widetilde{\bar{X}}_{\boldsymbol{a}}\left(\mathbb{F}_{p}\right)
$$

for some integer $h$.

LEMma 6.7. The numbers $\# \widetilde{\bar{X}}_{\boldsymbol{a}}\left(\mathbb{F}_{p}\right)$ and trace $\left(\operatorname{Frob}_{p} \mid H_{e ́ t}^{3}\left(\widetilde{\bar{X}}_{\boldsymbol{a}} \otimes \overline{\mathbb{F}}_{p}\right)\right)$ are even for primes $p$ of good reduction.

Proof. Equation (37) in Lemma 6.4 implies that $\#\left(\widetilde{\bar{X}}_{\boldsymbol{a}} \backslash T\right)\left(\mathbb{F}_{p}\right)$ is even. On $X_{\boldsymbol{a}} \cap T$ there is an involution $X_{i} \mapsto a_{i} / X_{i}$. The fixed points are exactly the singularities of $X_{\boldsymbol{a}}$ in $T$. After blowing up, these are replaced by a quadratic $Q$, with $\# Q\left(\mathbb{F}_{p}\right)=(p+1)^{2}$ or $p^{2}+1$ by Corollary 3.21. In either case this is an even number and so $\widetilde{\bar{X}}_{\boldsymbol{a}}\left(\mathbb{F}_{p}\right)$ is even. Now (39) implies that $\operatorname{trace}\left(\operatorname{Frob}_{p} \mid H_{\text {ét }}^{3}\left(\widetilde{\bar{X}}_{\boldsymbol{a}} \otimes \overline{\mathbb{F}}_{p}\right)\right)$ is also even. 
(L.1b) The cuspidal Hecke eigen forms we are interested in are $f_{6}, f_{12}$, $g_{30}, f_{30}, f_{30}^{\prime}, f_{60}$, and $f_{90}$, with $q$ expansions starting as in (1), (2), (6), (5), (7), (3) and (8). Arbitrarily many coefficients of the $q$-expansion of these forms may be computed by Stein's MAGma package [BCP], [St].

LEMMA 6.8. If $f$ is a cuspidal Hecke eigen form of level $N$ coprime to $2,3,5,7$, with q-expansion $f=\sum_{n>1} a_{n} q^{n}$, and if $a_{p}$ is even for primes $p$ with $11 \leq p \leq 37$, then $a_{p}$ is even for all primes $p \geq 11$.

Proof. This is proved by the same method as [Li, Proposition 4.10]. Tables of [J], list all $C_{3}$ and $S_{3}$ extensions of $\mathbb{Q}$ unramified outside 2, 3, 5, 7, and one can easily compute to see that the Frobenius at $p$ acting on any of these extensions has order 3 for at least one prime $p$ with $11 \leq p \leq 37$.

From this and the computation of enough terms of the $q$ expansions, we immediately obtain the following result.

Corollary 6.9. For a prime $p \neq 2,3,5,7$, the coefficient of $q^{p}$ in the $q$-expansion of the modular forms given by $f_{6}, f_{12}, g_{30}, f_{30}, f_{30}^{\prime}, f_{60}$, and $f_{90}$ are all even.

(L.1c) and (L.3b). This is a well known consequence of Poincaré duality. Let $V$ be a two dimensional piece of $H^{3}$ invariant under the Frobenius homomorphism. There is a map $\bigwedge^{2} V \rightarrow H^{6}$, which is nonzero by Poincaré duality. Since both spaces are 1-dimensional, this is an isomorphism. It follows from the fact that Frob $_{p}$ acts by multiplication by $p^{3}$ on $H^{6}$ that the determinant of the action on $H^{3}$ is $p^{3}$.

(L.2) By [Li, Proposition 4.11 b], when $\widetilde{\bar{X}}_{\boldsymbol{a}}$ has smooth reduction for $p \neq 2,3,5$, we can take

$$
T_{\{2,3,5\}}=\{7,11,13,17,19,23,29,31,41,43,53,61,71,73\} .
$$

Note that we can replace $7,11,13$ here by 103, 59, 37 respectively. This will become important later on when we shall determine the trace of Frobenius on $H_{\text {ét }}^{3}\left(\widetilde{\bar{X}}_{\boldsymbol{a}}\right)$. If $\widetilde{\bar{X}}_{\boldsymbol{a}}$ has bad reduction at $2,3,5,7$, then we use the following easy lemma.

Lemma 6.10. The elements of $\operatorname{Gal}(\mathbb{Q}[\sqrt{-1}, \sqrt{2}, \sqrt{3}, \sqrt{5}, \sqrt{7}] / \mathbb{Q})$ are given by the identity, together with Frob $_{p}$ for $p$ in the set

$$
\begin{aligned}
& T_{\{2,3,5,7\}}:=\{11,13,17,19,23,29,31,37,41,43,47,53,59,61,71,73,79,83, \\
&101,103,107,109,113,127,173,193,211,241,281,283,311\} .
\end{aligned}
$$




$\begin{array}{cccccccc}p & \# X_{1} & \# X_{9} & \# X_{4,4} & \# X_{4,4,9} & \# X_{25} & \# X_{9,9,9} & \# X_{4,4,4,16} \\ 7 & 3720 & 3160 & 3360 & 3172 & 3000 & 3092 & 3120 \\ 11 & 9240 & 7920 & 8424 & 7956 & 7464 & 7680 & 7848 \\ 13 & 13080 & 11260 & 12036 & 11368 & 10500 & 10940 & 11088 \\ 17 & 23400 & 20340 & 21420 & 20112 & 18540 & 19464 & 19920 \\ 19 & 29640 & 25840 & 27480 & 25840 & 24720 & 25352 & 25416 \\ 23 & 45120 & 39600 & 41904 & 39840 & 37560 & 38796 & 39144 \\ 29 & 76560 & 67860 & 71604 & 67584 & 65100 & 66408 & 66984 \\ 31 & 89400 & 79480 & 83376 & 79528 & 74664 & 76760 & 77880 \\ 41 & 172200 & 154980 & 161820 & 155172 & 148884 & 151632 & 153744 \\ 43 & 193080 & 174160 & 181224 & 174400 & 167640 & 170636 & 172656 \\ 53 & 320400 & 291780 & 303012 & 292392 & 281580 & 287112 & 289512 \\ 61 & 454440 & 416620 & 430788 & 416500 & 403884 & 408836 & 412608 \\ 71 & 663840 & 612720 & 634320 & 613032 & 592944 & 603720 & 609168 \\ 73 & 712920 & 658900 & 680700 & 658684 & 636180 & 647660 & 654048 \\ 103 & 1735320 & 1628200 & 1671168 & 1627156 & 1586040 & 1608716 & 1616976 \\ 59 & 418440 & 383040 & 397224 & 383124 & 367560 & 375720 & 378600 \\ 37 & 134760 & 120700 & 126804 & 121168 & 114900 & 118028 & 119808\end{array}$

Table 5: Number of points on $\widetilde{\bar{X}}_{\boldsymbol{a}}\left(\mathbb{F}_{p}\right)$, where for a sequence $\boldsymbol{b}=b_{1}, \ldots, b_{i}$ of length $i<6, X_{\boldsymbol{b}}$ means $\tilde{\bar{X}}_{\boldsymbol{a}}$, where $\boldsymbol{a}=\left(b_{1}: \cdots: b_{i}: 1: \cdots: 1\right)$.

Note that in $T_{\{2,3,5,7\}}$ we may replace 11 and 13 by 179 and 157 respectively.

(L.3a) Using the formula of Lemma 6.5, we obtain the data in Table 5.

From these values we shall be able to compute trace $\left.\operatorname{Frob}_{p}\right|_{V \boldsymbol{a}}$, where $V_{\boldsymbol{a}}$ is the two dimensional Galois representation given by $H_{\text {ét }}^{3}\left(\widetilde{\bar{X}}_{\boldsymbol{a}} \otimes \overline{\mathbb{F}}_{p}\right)$ in the rigid case, or by the subrepresentation given in Corollary 5.12 for the three nonrigid cases.

In the nonrigid cases, the elliptic curves $\mathcal{E}_{1,1,1,25}, \mathcal{E}_{1,9,9,9}$ and $\mathcal{E}_{4,4,4,16}$ have $j$-invariants $11^{3} 1259^{3} 2^{-1} 3^{-3} 5^{-4}, 11^{3} 13^{3} 23^{3} 2^{-1} 3^{-12} 5^{-1}$ and $71^{3} 2^{-4}$. $3^{-3} 5^{-1}$ respectively, but they all have conductor 30 . They are isogenous to each other, since there is only one weight 2 level 30 Hecke eigen newform, $g_{30}=\sum b_{n} q^{n}$, with $b_{p}$ for primes $p \in T_{2,3,5}$ (see (40)) as in the following 


$\begin{array}{cccccccc}p & V_{1} & V_{9} & V_{4,4} & V_{4,4,9} & V_{25} & V_{9,9,9} & V_{4,4,4,16} \\ 17 & -126 & -126 & 18 & 102 & 42 & -66 & -114 \\ 19 & 20 & 20 & -100 & 20 & -76 & -100 & 140 \\ 23 & 168 & 168 & 72 & -72 & 0 & -132 & 72 \\ 29 & 30 & 30 & -234 & 306 & 6 & 90 & 210 \\ 31 & -88 & -88 & -16 & -136 & -232 & 152 & 272 \\ 41 & 42 & 42 & 90 & -150 & 234 & 438 & -198 \\ 43 & -52 & -52 & 452 & -292 & -412 & 32 & -268 \\ 53 & 198 & 198 & 414 & -414 & 222 & -222 & -78 \\ 61 & -538 & -538 & 422 & -418 & -490 & 902 & 302 \\ 71 & 792 & 792 & -360 & 480 & 120 & -432 & -768 \\ 73 & 218 & 218 & 26 & 434 & 746 & 362 & -478 \\ 103 & 128 & 128 & 8 & 1172 & -560 & -1812 & 640 \\ 59 & -660 & -660 & -684 & -744 & 660 & -420 & 240 \\ 37 & 254 & 254 & -226 & -214 & 430 & 114 & -260 \\ \text { level } & 6 & 6 & 12 & 60 & 30 & 90 & 30\end{array}$

Table 6: Values of trace $\left(\left.\operatorname{Frob}_{p}\right|_{V_{a}}\right)$.

table.

$$
\begin{array}{ccccccccccccccc}
p & 7,103 & 11,59 & 13,37 & 17 & 19 & 23 & 29 & 31 & 41 & 43 & 53 & 61 & 71 & 73 \\
b_{p} & -4 & 0 & 2 & 6 & -4 & 0 & -6 & 8 & -6 & -4 & -6 & -10 & 0 & 2
\end{array}
$$

Now from (29) and (39), we have

$$
\operatorname{trace}\left(\left.\operatorname{Frob}_{p}\right|_{V_{\boldsymbol{a}}}\right)=p^{3}+1+p(1+p) h-\# \widetilde{\bar{X}}_{\boldsymbol{a}}\left(\mathbb{F}_{p}\right)-h^{12}\left(\widetilde{\bar{X}}_{\boldsymbol{a}}\right) p b_{p} .
$$

From this we see that using the data in Tables 1 and 5 we can compute the values of trace $\left(\left.\operatorname{Frob}_{p}\right|_{V_{a}}\right)$ provided we know the integer $h$. At this point we make use of a trick which to our knowledge goes back to van Geemen and Werner. Recall that by the analogue of the Riemann hypothesis the absolute value of the trace of Frobenius on an invariant 2-dimensional piece of $H_{\text {ét }}^{3}\left(\widetilde{\bar{X}}_{\boldsymbol{a}}\right)$ is bounded by $2 p^{3 / 2}$. If $p \geq 17$ then the value of $p(1+p)$ exceeds $4 p^{3 / 2}$ and hence $h$ and thus also trace $\left(\left.\operatorname{Frob}_{p}\right|_{V_{\boldsymbol{a}}}\right)$ can be determined by the above formula. Using this observation we obtain values as in Table 6, where we use the same indexing convention as in Table 5.

Note that in all of these cases $h=h^{11}\left(\widetilde{\widetilde{X}}_{\boldsymbol{a}}\right)$. We conjecture that this is always the case. To prove this, it would be enough to prove that the action 


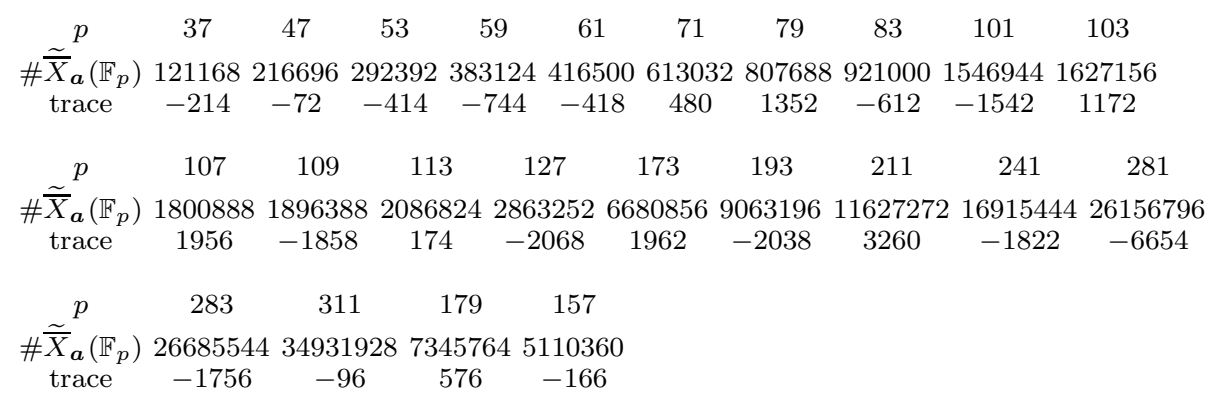

Table 7: Values of $\# \widetilde{\bar{X}}_{\boldsymbol{a}}\left(\mathbb{F}_{p}\right)$ and $\operatorname{trace}\left(\left.\operatorname{Frob}_{p}\right|_{V_{\boldsymbol{a}}}\right)$ for $\boldsymbol{a}=(1: 1: 4: 4: 9)$.

of the Frobenius on $H^{2}$ and $H^{4}$ is multiplication by $p$ and $p^{2}$ respectively. The above numbers can be verified to be coefficients of weight 4 cuspidal Hecke eigen modular forms of levels 6, 6, 12, 60, 30, 90 and 30 respectively, as indicated in the last column. In Lemma 6.2 we saw that $\widetilde{X}_{(1: 1: 4: 4: 9)}$ also has bad reduction at 7 . Thus in this case we have to compute the number of point over $\mathbb{F}_{p}$ for additional primes as given in Lemma 6.10, and from this, as before, we can compute the values of the traces on $V_{\boldsymbol{a}}$. This data is given in Table 7 .

Thus, applying [Li, Theorem 4.3], we have the following.

Theorem 6.11. For $\boldsymbol{a}$ as in Corollaries 4.9 and 5.12 the two dimensional Galois representations $V_{\boldsymbol{a}}$ given by (28) are modular, corresponding to the weight 4 modular forms, with coefficients and level indicated in Table 6 .

\section{REFERENCES}

[Ba] V. V. Batyrev, Dual polyhedra and mirror symmetry for Calabi-Yau hypersurfaces in toric varieties, Journal of Algebraic Geometry, 3 (1994), 493-535.

[Bo] C. Borcea, Polygon spaces, tangents to quadrics and special Lagrangians, Oberwolfach Report, 42 (2004), 2181-2183.

[BCDT] C. Breuil, B. Conrad, F. Diamond and R. Taylor, On the modularity of elliptic curves over $\mathbb{Q}$ : wild 3-adic exercises, J. Am. Math. Soc., 14 (2001), no. 4, 843-939.

[BCP] W. Bosma, J. Cannon and C. Playoust, The Magma algebra system. I. The user language, J. Symbolic Comput., 24 (1997), no. 3-4, 235-265.

[C] H. Clemens, Double solids, Adv. in Math., 47 (1983), 107-230. 
[CS] C. Consani and J. Scholten, Arithmetic on a quintic threefold, International Journal of Mathematics, 12 (2001), no. 8, 943-972.

[CM] S. Cynk and C. Meyer, Geometry and Arithmetic of certain Double Octic Calabi-Yau Manifolds, preprint, math.AG/0304121, Canad. Math. Bull., 48 (2005), no. 2, 180-194.

[DM] L. Dieulefait and J. Manoharmayum, Modularity of rigid Calabi-Yau threefolds over $\mathbb{Q}$, Calabi-Yau varieties and mirror symmetry (Toronto, ON, 2001) (N. Yui and J. D. James, eds.), Fields Institute Communications 38, Amer. Math. Soc., Providence, RI (2003), pp. 159-166.

[DL] I. Dolgachev and V. Lunts, A character formula for the representation of a Weyl group in the cohomology of the associated toric variety, J. Algebra, 168 (1994), no. $3,741-772$.

[EH] D. Eisenbud and J. Harris, The geometry of schemes, Graduate Texts in Mathematics 197, Springer-Verlag, New York, 2000.

[FM] J.-M. Fontaine and B. Mazur, Geometric Galois representations, Elliptic curves, modular forms, \& Fermat's last theorem, Series in Number Theory, I, Internat. Press, Cambridge, MA (1995), pp. 41-78.

[FK] E. Freitag and R. Kiehl, Ètale cohomology and the Weil conjecture, Ergeb. Math. Grenzgeb. (3) 3, Springer Verlag, 1988.

[F] W. Fulton, Intersection Theory, Second Edition, Springer Verlag, 1998.

$[\mathrm{GW}] \quad$ B. van Geemen and J. Werner, Nodal quintics in $\mathbb{P}^{4}$, Arithmetic of complex manifolds, Proceedings Erlangen 1988 (W.-P. Barth and H. Lange, eds.), Springer Lecture Notes 1399 (1989), pp. 48-59.

[Ha] R. Hartshorne, Algebraic geometry, Graduate Texts in Mathematics 52, Springer-Verlag, New York, 1977.

[H] J. W. P. Hirschfeld, Projective geometries over finite fields, Oxford Mathematical Monographs, The Clarendon Press, Oxford University Press, New York, 1979.

[Ko] J. Kollár, Flops, Nagoya Math. J., 113 (1989), 15-36.

[J] J. Jones, Tables of number fields with prescribed ramification. http://math.la.asu.edu/ jj/numberfields/.

[Li] R. Livné, Cubic exponential sums and Galois representations, Contemporary Mathematics, 67 (1987), 247-261.

[LY] R. Livné and N. Yui, The modularity of certain non-rigid Calabi-Yau threefolds, math.AG/0304497, to appear, J. Math. Kyoto Univ.

[Lu] K. Ludwig, Torische Varietäten und Calabi-Yau-Mannigfaltigkeiten, Diplomarbeit, Institut für Mathematik, Universität Hannover (2003).

(available from http://www-ifm.math.uni-hannover.de/ ${ }^{\sim h u l e k / A G / d a t a / ~}$ DiplomarbeitLudwig.pdf).

[P] C. Procesi, the toric variety associated to the Weyl chambers, in "mots" (M. Lothaire, ed.), Hermés, Paris (1990), pp. 153-161.

[Se1] J.-P. Serre, Cours d'arithmétique, Deuxiéme édition revue et corrigée, Le Mathématicien, No. 2, Presses Universitaires de France, Paris, 1977.

[Se2] J.-P. Serre, Représentations l-adiques, Kyoto Int. Symposium on algebraic num- 
ber theory, Japan Soc. for the Promotion of Science (1977), pp. 177-193. (oeuvres, t. III, 384-400).

[Sc] C. Schoen, On fiber products of rational elliptic surfaces with section, Math. Z., 197 (1988), no. 2, 177-199.

[St] W. A. Stein, Explicit approaches to modular abelian varieties, U. C. Berkeley Ph.D. thesis (2000).

[V] H. A. Verrill, Root lattices and pencils of varieties, J. Math. Kyoto Univ., 36 (1996), 423-446.

[We] J. Werner, Kleine Aufösungen spezieller dreidimensionaler Varietäten, Bonner Mathematische Schriften 186, 1987.

[Wi] A. Wiles, Modular elliptic curves and Fermat's Last Theorem, Ann. Math. (2), 141 (1995), no. 3, 443-551.

[Y1] N. Yui, The arithmetic of certain Calabi-Yau varieties over number fields, The arithmetic and geometry of algebraic cycles (Banff, AB, 1998), NATO Sci. Ser. C, Math. Phys. Sci., 548, Kluwer Acad. Publ., Dordrecht (2000), pp. 515-560.

[Y2] N. Yui, Update on the modularity of Calabi-Yau varieties, With an appendix by Helena Verrill, Calabi-Yau varieties and mirror symmetry (Toronto, ON, 2001) (N. Yui and J. D. James, eds.), Fields Institute Communications 38, Amer. Math. Soc., Providence, RI (2003), pp. 159-166.

Klaus Hulek

Institut für Mathematik ( $C)$

Universität Hannover

Welfengarten 1

30060 Hannover

Germany

hulek@math . uni-hannover.de

Helena Verrill

Department of Mathematics

Louisiana State University

Baton Rouge, LA 70803-4918

U.S.A.

verrill@math.lsu.edu 